\title{
ON DISCRETE STRUCTURE OF GEOLOGIC MEDIUM AND CONTINUAL APPROACH TO MODELING ITS MOVEMENTS
}

\author{
Sh. A. Mukhamediev
}

O.Yu. Schmidt Institute of Physics of the Earth of RAS, Moscow, Russia

\begin{abstract}
This paper discusses the structure of a geologic medium represented by accessible lithified rocks and provides an overview of methods used to describe its movements. Two basic opinions are considered in the framework of the discussion: (1) an initially homogeneous and continuous geologic medium acquires the structure composed of blocks in the process of the geologic medium's deformation/destruction/degradation, and (2) a geologic medium is composed of blocks (and often has hierarchic, active, energy-saturated features), and the continuity model is thus not valid for describing the geologic medium's deformation. Proponents of the first point of view actively apply the standard or modified continuum model of a solid deformed body (SDB) in estimations of the stress-strain state, but the input parameters of this model do not contain any information on discreteness in principle. Authors who support the second opinion, either explicitly or implicitly assume that the block structure of the geologic medium, which is detectable by geological methods, makes a direct and unambiguous impact on all other mechanical properties of the geologic medium and, above all, on the nature of its movements.

Based on results obtained by interpreting the data collected in our long-term field studies of rock fracturing, mathematical processing of GPS-measurements, and theoretical models, we agree with the concept of the geologic medium's block structure, but argue that the geologic block-structure property is not acquired but congenital. Regarding sedimentary rocks, it means that the discrete structure has been already embodied in the rock before sediment lithification, regardless of the intensity of macroscopic deformations. A discrete structure is the form of the geologic medium existence and a cause of the congenital anisotropy of the geologic medium's strength characteristics. Due to subsequent deformation of the geologic medium, some elements of the structure can be manifested more clearly, and the structure itself can become more complex due to secondary effects. At the same time, the structure of geologic blocks is not directly manifested in the spatial and temporal features of the recent movements in the geologic medium. However, it is not an obstacle to developing continuum models of such movements in the same way as, for instance, the adequacy of the continuum general theory of relativity is not denied in view of the discrete-hierarchical structure of the Universe.
\end{abstract}

The key requirements to a model include its applicability, testability, confirmability/deniability of its predictions in the investigated space-time scale, and compliance with conservation laws. This paper briefly discusses the most important aspects of the continuum approach based on the concept of an effective continuous medium and, above all, the Cauchy continuum model, envisaging that the dynamic response of the medium in spatial descriptions is given only by the Cauchy symmetric stress tensor (T). In more general continuum models of the medium (such as moment, micropolar, micromorphic and other models), the dynamics of the medium may be characterized by asymmetric tensors of force and couple stresses.

This paper refutes the unjustified criticism of the continuum model as such criticism is rooted in the mistaken identification of quite special assumptions or ways of setting the problems with the general principles of the continuity model. Special attention is given to critical comments received from supporters of the active geologic medium concept. The paper considers actual difficulties encountered in studies using the continuity model, specifically in coordinated descriptions of the medium containing mobile defects, as well as the medium that is subject to deformation due to movements on its structure, while this structure is hierarchical at any scale level, down to the zero level (which, in particular, concerns the fractal structure). Discussed are causes of some widespread misunderstandings and mistakes in the geoscience literature, as well as the occurrence of conditions facilitating the revival of Aristotle ideas and preNewton concepts in geology, which repeatedly gain the upper hand over the modern ideas of classical physics. The paper considers the problem of reconstruction of stresses in the geologic medium from in-situ kinematic indicators, specifically from irreversible slips. Attention is drawn to the fact that the currently dominating approach 'imposes' a priori speculative rules on the geologic medium, such as a relationship between stresses (to be estimated) and the slip directions. Under this approach, the conservation laws are inevitably ignored, which makes it impossible to interpret the obtained results in terms of stress. Under the alternative approach proposed earlier by the author of this paper, the conservation laws being taken into account allows not only to reconstruct the stress tensor field, but also to judge 
on the geologic medium rheology. It is concluded that rejecting the continuum approach a priori, with a reference to the geologic medium discreteness, is at least unconstructive.

Key words: geologic medium; block structure; continuity; stress; deformation; fracture; conservation laws

\title{
Recommended by S.I. Sherman
}

For citation: Mukhamediev Sh.A. 2016. On discrete structure of geologic medium and continual approach to modeling its movements. Geodynamics \& Tectonophysics 7 (3), 347-381. doi:10.5800/GT-2016-7-30213.

Для цитирования: Мухамедиев Ш.А. О дискретном строении геосреды и континуальном подходе к моделированию ее движения // Геодинамика и тектонофизика. 2016. Т. 7. № 3. С. 347-381. doi: 10.5800/GT-2016-7-3-0213.

\section{О ДИСКРЕТНОМ СТРОЕНИИ ГЕОСРЕДЫ И КОНТИНУАЛЬНОМ ПОДХОДЕ К МОДЕЛИРОВАНИЮ ЕЕ ДВИЖЕНИЯ}

\section{Ш. А. Мухамедиев}

\author{
Институт физики Земли им. О.Ю. Шмидта РАН, Москва, Россия
}

Аннотация: Настоящая работа посвящена обсуждению строения геосреды, т.е. доступных для изучения литифицированных горных пород, и способов описания движения этой среды. В рамках обсуждаемых вопросов существуют два мнения: 1) изначально однородная сплошная геосреда приобретает свойство блочности в процессе ее деформирования (а также деструкции, деградации, разрушения) и 2) геосреда является блочной (а нередко, помимо этого, иерархической, активной, энергонасыщенной), и поэтому модель сплошности недопустима при описании ее деформирования. Сторонники первой точки зрения активно применяют стандартную или модифицированную континуальную модель твердого деформируемого тела (ТДТ) в расчетах напряженно-деформированного состояния, но входные параметры модели принципиально не содержат сведения о дискретности. Авторы, придерживающиеся второго мнения, явно или неявно полагают, что блочная структура геосреды, выявляемая геологическими методами, прямым и однозначным образом влияет на все ее иные механические свойства и, в первую очередь, на характер ее движения.

Опираясь на интерпретацию данных, полученных в ходе многолетних полевых исследований трещиноватости горных пород, математическую обработку результатов GPS-измерений и построенные теоретические модели, мы соглашаемся с тезисом о блочном строении геосреды, но утверждаем, что свойство геологической блочности является не приобретенным, а врожденным. В отношении осадочных горных пород это означает, что дискретная структура запечатлена в породе еще до момента ее зарождения из осадков (литификации) независимо от интенсивности макроскопических деформаций. Дискретная структура является формой существования геосреды и причиной врожденной анизотропии ее прочностных характеристик. При последующих деформациях геосреды те или иные элементы структуры выявляются четче, сама структура может обогатиться за счет вторичных эффектов.

В то же время структура геологических блоков не проявляется прямым образом в пространственновременных особенностях современных движений геосреды и не служит препятствием к построению континуальных моделей этих движений подобно тому, как, например, дискретно-иерархическая структура Вселенной не отрицает адекватности континуальной общей теории относительности. Важнейшими требованиями к модели являются ее применимость и возможность подтверждения или опровержения ее предсказаний на исследуемом пространственно-временном масштабе, а также выполнение в рамках модели законов сохранения. В работе кратко рассматриваются важнейшие аспекты континуального подхода, использующего концепцию эффективной сплошной среды, и, в первую очередь, модель континуума Коши, в которой динамический отклик среды при пространственном описании задается единственным силовым симметричным тензором напряжений Коши Т. В рамках более общих континуальных моделей среды (моментных, микрополярных, микроморфных и т.д.) динамику среды могут характеризовать несимметричные тензоры силовых и моментных напряжений.

В статье опровергается неоправданная критика континуальной модели, которая имеет своими корнями отождествление весьма частных предположений или способов постановки задач с общими принципами модели сплошности. Определенное внимание уделяется критике, высказываемой приверженцами концепции активной геосреды. Рассматриваются те действительные трудности, которые встречаются в модели сплошности при отсчетном описании среды с мобильными объемными дефектами, а также среды, деформирование которой происходит вследствие движений по структуре, иерархической на любом масштабном уровне вплоть до нулевого размера, в частности по фрактальной структуре. Обсуждается генезис некоторых типичных ошибок в геолого-геофизической литературе и причины возникновения благоприятных условий для 
того, чтобы в геологии воззрения Аристотеля и другие доньютоновские представления раз за разом возрождались и одерживали верх над идеями современной классической физики. Рассмотрена проблема реконструкции напряжений в геосреде по натурным кинематическим индикаторам, а именно по необратимым разрывным сдвигам. Обращается внимание на то, что в господствующем сейчас подходе авторы пытаются «навязать» геосреде некоторые априорные умозрительные правила связи искомых напряжений с кинематикой подвижек. В рамках такого подхода законы сохранения неизбежно игнорируются, что не дает возможности интерпретировать получаемые результаты в терминах напряжений. Альтернативный подход, предложенный ранее автором данной работы, позволяет с учетом законов сохранения не только реконструировать поле тензора напряжений, но и, дополнительно к этому, судить о реологии геосреды. В заключение статьи утверждается, что отвергать континуальный подход априори, ссылаясь на дискретность геосреды, по меньшей мере неконструктивно.

Ключевые слова: геосреда; блочность; сплошность; напряжение; деформация; трещина; законы сохранения

\section{ОГЛАВЛЕНИЕ}

1. Введение 349

2. Характер движения геосреды и модели сплошности ................................................................................ 351

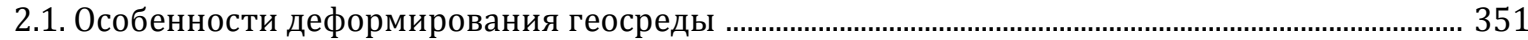

2.2. О противопоставлении строения геосреды и моделей описания ее движения ....................... 352

2.3. Примеры континуального подхода к описанию дискретной и дискретно-иерархической сред ………............................................................................................. 353

3. Элементы классической модели твердого деформируемого тела .................................................... 354

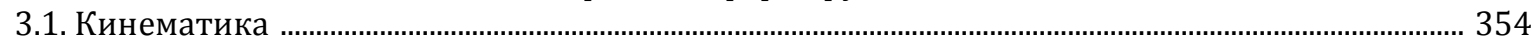

3.2. Напряжения и законы сохранения для континуума Коши ........................................................... 355

3.3. Определяющие соотношения в континууме Коши …......................................................................... 356

4. Учет микроструктуры и обобщения континуума Коши ……................................................................. 357

4.1. Способы прямой континуализации дискретной среды .................................................................... 358

4.2. Об обобщениях континуума Коши ............................................................................................................ 358

4.3. О континуализации искусственной и естественной блочной структуры .................................. 359

5. Модели возникновения блочной структуры геосреды ……..................................................................... 360

5.1. Представления о приобретенной дискретности ................................................................................. 360

5.2. Представления о врожденной дискретности …....................................................................................... 361

6. Опровержение критики континуальной модели ..................................................................................3 362

6.1. О критике со стороны последователей Л.Д. Ландау и Е.М. Лившица ............................................. 362

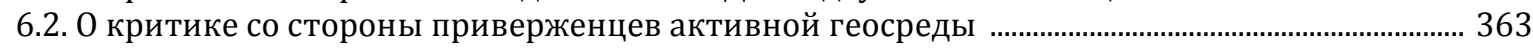

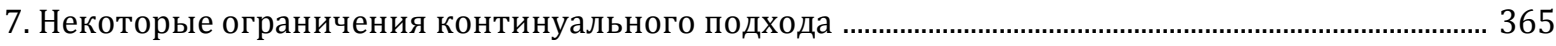

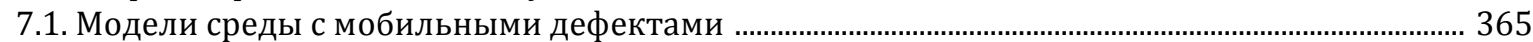

7.2. Модели среды с иерархическим движением на любом уровне ................................................... 367

8. Генезис типичных ошибок и неиспользованные возможности континуального

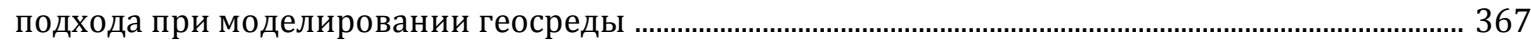

8.1. О терминах и понятиях, заимствованных геологией из физики ................................................. 367

8.2. Генезис воспроизводства доньютоновских воззрений в геологии .............................................. 368

8.3. Игнорирование физики в методах реконструкции напряжений по кинематическим данным ....................................................................................................................... 370

8.4. Об учете законов сохранения в методах реконструкции напряжений по

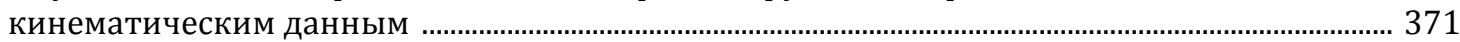

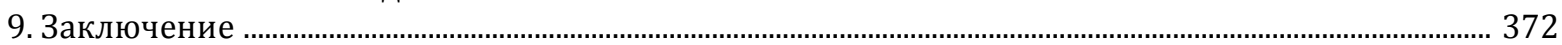

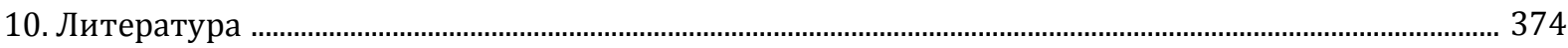

\section{1. ВВЕДЕНИЕ}

Спор между сторонниками дискретности и приверженцами сплошности природной среды является одной из наиболее древних научных дис- куссий в истории цивилизации. В Европе эта дискуссия восходит ко временам древних греков. Еще в V в. до н.э. Левкипп и Демокрит (а вслед за ними Эпикур, Лукреций и другие) отстаивали мнение об атомистической природе материи. Не следует ду- 
мать, что под атомами античные мыслители непременно подразумевали мельчайшие частицы вещества. Имелись в виду неделимые части, что соответствует переводу термина «атом» с древнегреческого. Например, Демокрит признавал существование атомов самой различной величины, в том числе и весьма больших размеров [Zubov, 1965]. Противники атомизма утверждали, что материя делится до бесконечности. Наиболее авторитетным приверженцем идеи сплошности был, безусловно, Аристотель (384-322 до н.э.). Защищая сплошность, Аристотель опирался на выдвинутую им модель движения, которая входила в конфликт с представлениями о наличии пустоты. С течением времени в дискуссии тип дискретности варьировался (корпускулы, молекулы, элементарные частицы, звездные системы). Иногда дискуссия достигала предельной остроты. Например, в 1624 г. Парижский парламент под страхом смертной казни запретил диспуты, в которых пропагандировалось атомистическое учение [Lurie, 1935].

В случае геосреды ${ }^{1}$ дискуссия приобретает специфические особенности. Изучая и интерпретируя экспериментальные данные, исследователи часто приходят к выводу о дискретном строении геосреды, о ее иерархической структуре. Это мнение отражается и на страницах журнала Geodynamics \& Tectonophysics (см., например [Sherman, 2012; Seminsky et al., 2013]). В то же время среди сторонников дискретности можно выделить две группы по критерию их представлений о моделировании геосреды.

Первую группу составляют авторы, исходящие из представления, согласно которому «доступное измерению вещество земной коры образует блочно-иерархическую структуру, являющуюся результатом процессов деструкции» [Goldin, 2002]. Часто сторонники подобных взглядов, говоря об изменяющихся свойствах геосреды, употребляют и термин «деградация». Согласно словарям иностранных слов, «деградация» (от французского dégradation) означает процесс изменения чего-либо в сторону ухудшения, утрату ранее накопленных свойств, а термин «деструкция» заимствован от латинского destructio, что означает нарушение нормальной структуры чего-либо [Lekhin, Petrov, 1949]. Что же в приведенном высказывании С.В. Гольдина подразумевается под нормальной структурой геосреды? Очевидно, это структура, ранее не

\footnotetext{
1 Говоря о геосреде, мы далее подразумеваем те части верхней оболочки Земли, которые доступны для наблюдений и экспериментального исследования и которые сложены твердыми (литифицированными) горными породами. Весьма условно геосреду, изучаемую методами геологии, можно назвать геологической средой, а изучаемую методами геофизики - геофизической.
}

имевшая приобретенных в «результате процессов деструкции» свойств блочности (дискретности) и иерархичности, т.е. сплошная макроскопически однородная и изотропная по прочности твердая горная порода. Конечно, весьма странно, что в качестве нормального назначается такое умозрительное состояние геосреды, которое никто никогда не наблюдал. Для дальнейшего важно, что авторы, придерживающиеся подобных представлений, в своих попытках теоретически смоделировать возникновение дискретности отталкиваются от образа однородной сплошной на макроскопическом уровне среды и принимают такое состояние за начальное.

Вторая группа состоит из авторов, априорно отрицающих возможность использования континуального подхода к моделированию геосреды. Их мнение можно сформулировать следующим образом: «Геосреда является дискретной (варианты: блочной, блочно-иерархической, энергонасыщенной, активной), поэтому использование модели сплошной среды для описания ее поведения сомнительно [Sadovsky, 1989], в принципе недопустимо [Vikulin, 2008], вызывает удивление [Koronovsky, Naimark, 2013]». Здесь первая часть утверждения отражает наблюдаемый факт и не вызывает у нас возражений. Впрочем ни у кого не вызывает возражения и факт дискретности вообще любого вещества, имеющего в естественных земных условиях атомно-молекулярное строение. Но на этом основании никто не выдвигает принципиальных возражений, скажем, против законов Бойля-Мариотта или Гука, сформулированных в рамках представлений о сплошности. Предметом дискуссии могут стать вопросы границ применимости этих законов, поэтому в вышеприведенном утверждении кроется некорректность, заключающаяся в следующем. Нельзя материальные объекты и их строение (т.е. данные наблюдений, так или иначе интерпретированные) противопоставлять теоретическим моделям движения этих объектов (т.е. способам описания возникающих деформаций и напряжений). Характерно то, что свое мнение о неадекватности континуальной модели ее противники подкрепляют, противопоставляя реальной среде лишь чрезвычайно упрощенные модели континуума, обычно модель безмоментного линейноупругого тела с нулевыми начальными напряжениями. Такая обедненная модель незаконно отождествляется с общей моделью континуума, что весьма облегчает критику последней. При этом, отвергая на словах модель сплошности, критики, как само собой разумеющимся, оперируют понятием напряжений, не уточняя, что они под этим подразумевают. Между тем понятие механического напряжения возникает лишь при континуализации 
среды, причем вопрос о том, что есть напряжение в той или иной модели среды, отнюдь не тривиален и в рамках некоторых моделей не решен однозначHO.

В настоящей работе, посвященной вопросам интерпретации наблюдаемого строения и теоретического моделирования движения геосреды, мы соглашаемся с тезисом о ее блочном строении, но, в отличие от авторов первой группы, утверждаем, что свойство геологической блочности является не приобретенным, а врожденным. В отношении осадочных горных пород это означает, что дискретная структура, выявляемая в породах одного и того же вещественного состава, запечатлена в этой породе еще до момента ее зарождения из осадков (литификации) независимо от интенсивности макроскопических деформаций. В то же время мы возражаем авторам второй группы и отрицаем неприменимость модели сплошности к описанию движения геосреды. В разделе 2 обсуждаются требования, предъявляемые к построению моделей, и приводятся примеры адекватного применения континуальной модели к описанию дискретных и иерархичных сред. В разделе 3 конспективно излагаются некоторые положения континуальной модели Коши. Судя по критике, высказываемой ортодоксальными ниспровергателями модели сплошности, они с этими положениями не вполне знакомы. В разделе 4 кратко отмечаются отдельные аспекты континуального подхода, выходящие за рамки модели Коши. Раздел 5 посвящен обсуждению вопросов возникновения блочной структуры, а раздел 6 опровержению той неоправданной критики континуальной модели, которая имеет своими корнями отождествление весьма частных предположений или способов постановки задач с общими принципами модели сплошности. В разделе 7 рассматриваются те действительные трудности, которые возникают в модели сплошности при отсчетном описании движения. И, наконец, в разделе 8 обсуждается генезис некоторых типичных ошибок в геолого-геофизической литературе и иллюстрируется важность соблюдения законов сохранения при моделировании геосреды.

\section{2. ХАРАКТЕР ДВИЖЕНИЯ ГЕОСРЕДЫ И МОДЕЛИ СПЛОШНОСТИ}

\section{1. ОСОБЕННОСТИ ДЕФОРМИРОВАНИЯ ГЕОСРЕДЫ}

Чтобы убедиться в блочном квазирегулярном строении геосреды, необязательно изучать чьилибо научные труды, достаточно поверить своим глазам, находясь на любом обнажении горных пород и убеждаясь в обязательном наличии систем трещин, делящих слои на блоки. В обнажениях осадочных пород можно заметить и иерархичность на смежных масштабах: наряду с внутрислойными трещинами существуют более редкие и более крупные трещины (так называемые master-cracks), секущие несколько слоев подряд и ориентированные (по крайней мере в условиях древних платформ) согласно простиранию систем внутрислойной трещиноватости. Удостоверившись в увиденном и в существовании крупных линейных элементов рельефа того же простирания, нетрудно согласиться с тезисом об иерархичности на нескольких масштабах. Картины регулярности дискретного строения в тектонически активных регионах могут затушевываться и искажаться из-за больших деформаций и наложенных вторичных структур.

Вследствие достаточно очевидного блочного строения верхних горизонтов земной коры извечный спор о дискретности и сплошности в случае геосреды в настоящее время заметно изменил свою направленность и нацелился на обсуждение ее движения. Здесь наиболее интенсивные дебаты сконцентрировались вокруг характера деформирования континентальной коры, который приобрел статус одной из важнейших проблем геодинамики [Thatcher, 2003]. Существуют две крайние точки зрения на решение проблемы в рамках активной тектоники. Согласно одному взгляду, деформация литосферы происходит за счет подвижек по границам дискретных микроплит - блоков (например [Avouac, Tapponnier, 2003]). Альтернативное представление исходит из того, что, напротив, деформация непрерывно распределена по объему (например [England, Molnar, 1997]). Дискуссия развивается в основном на материале азиатских регионов, в той или иной мере затронутых процессом коллизии Индийской и Евразийской плит. Количественно критерий адекватности каждой из точек зрения не формализован. Считается, что при уменьшении размеров блоков и увеличении числа разломов блочная модель становится все ближе к континуальной [England, Molnar, 2005; Thatcher, 2007].

Попытка непосредственного выявления блочной структуры в поле современных горизонтальных скоростей по данным Центрально-Азиатской GPS-сети [Mukhamediev et al., 2006; Kuzikov, Mukhamediev, 2010] показала, что упрощенная альтернатива «дискретность-сплошность», представленная упомянутыми выше точками зрения, а также мнение о возможном способе их сближения нуждаются, по меньшей степени, в серьезной корректировке, если речь идет о современных движениях. Выделенные односвязные квазижесткие 2D домены, существующие в настоящий момент и испытыва- 
ющие поступательные и вращательные движения, не контактируют между собой по границам («разломам»), а разделены относительно широкими зонами повышенных деформаций. Пространственная корреляция между этими зонами и геологическими разломами отсутствует, хотя существует взаимосвязь между их угловыми распределениями и направлениями сдвигов по ним. В геометрии и кинематике выявленных зон прослеживается скрытая регулярность, отражающая динамическое воздействие Индийской плиты [Kuzikov, Mukhamediev, 2010; Mukhamediev et al., 2011].

По данным той же GPS-сети в рамках континуальной модели было рассчитано поле градиента скорости современных горизонтальных движений коры [Zubovich, Mukhamediev, 2010]. Результаты обнаружили наличие в исследуемом поле неоднородностей, существенно отличающихся по интенсивности деформаций и вращений от фоновых значений. Сравнение результатов континуальных и дискретных расчетов [Mukhamediev et al., 2011] показало, что, за исключением мелких деталей, согласуются как общая картина вращений, так и крупные элементы структурированности поля деформаций. Видимо, скрытая регулярность деформаций, выявляемая обеими моделями, - устойчивое во времени свойство, порожденное длительным внешним воздействием, чего не скажешь о блоковой структуре скоростей. Вопреки устоявшимся представлениям об обязательно точном и однозначном отражении дискретности, выявляемой геологическими методами в характере деформирования геосреды, выделенные по данным о GPS-скоростях блоки никак не соответствуют геологическим блокам. Конфигурация и параметры движения этой современной блочной структуры, возникающей в поле градиента скорости, изменчивы и зависят, как минимум, от периода наблюдений. Они могут измениться, например, после одного или нескольких землетрясений вследствие того, что участки геологических разломов, «молчавшие» за период GPS-измерений ( $\approx 10$ лет) и входившие в состав блоков, активизируются и перейдут в зоны повышенных деформаций. Отмеченное несоответствие блочных структур показывает, что строение геологической среды и структурные особенности движения геофизической среды не являются тождественными понятиями.

\section{2. О ПРОТИВОПОСТАВЛЕНИИ СТРОЕНИЯ ГЕОСРЕДЫ И МОДЕЛЕЙ ОПИСАНИЯ ЕЕ ДВИЖЕНИЯ}

Во Введении отмечался факт противопоставления некоторыми авторами строения геосреды и моделей описания ее движения. В связи с этим полезно вспомнить афоризм американского стати- стика Дж. Бокса (1978): «Все модели неверны, но некоторые - полезны» [Mora, 2014]. Задолго до этого афоризма мысль о неизбежности и необходимости разумных упрощений при моделировании сложных природных явлений высказывалась многими исследователями, начиная с древнегреческих. Но для разных областей знания эта мысль воплощается в практику неодинаково. У исследователя с геологическим типом мышления ${ }^{2}$ (в дальнейшем геолога) по сравнению с физиком баланс между учетом подробностей явления и абстрагированием от таковых заметно смещен в пользу подробностей. Однако ценность модели определяется отнюдь не тем, насколько она верна, т.е. насколько подробно она копирует действительность. Согласно датскому математику Г. Рашу (1960), «модели не обязаны быть верными, важно, чтобы они были применимыми» [Mora, 2014]. Естественно, что применимость (и работоспособность) модели для достижения поставленных целей должна быть исследована. История науки знает случаи применимости моделей, которые с позиций современных знаний представляются заведомо неверными. К их числу относятся модель теплорода, позволившая Фурье вывести закон теплопроводности, и механическая (шестеренчатая) модель эфира, опираясь на которую Максвелл получил уравнения электромагнитного поля. История науки также показывает, что под работоспособные уравнения впоследствии будет подведен более надежный теоретический фундамент.

Рассматривая неоднородную геосреду, исследователи обычно выдвигают следующий критерий возможности использования континуального описания:

$$
d \ll l \ll L,
$$

где $L, l$ и $d$ - характерные линейные размеры исследуемой системы, репрезентативного элемента среды (см. раздел 3) и неоднородности. Соответственно, нарушение этого критерия для геосреды считается достаточным основанием для отказа континуальному подходу в праве описания этой среды (например [Koronovskii, Naimark, 2015]). По нашему мнению, догматическое использование критерия (1) в качестве априорного запретительного инструмента является ошибочным методологическим приемом по разным причинам.

\footnotetext{
2 Под геологическим типом мышления мы подразумеваем метод познания, основанный на индукции, т.е. на переходе от частного знания к общему. Этот метод связан с обобщением результатов наблюдений и экспериментов, он помогает искать истину, но не обязательно гарантирует ее достижение. Точным наукам более присущ метод дедукции, т.е. переход в процессе познания от общего знания о некотором классе предметов и явлений к знанию частному и единичному. Методы индукции и дедукции являются скорее взаимодополняющими, чем взаимоисключающими.
} 
1. Критерий (1) неявно подразумевает необходимость копирования строения среды, т.е. «допускает к конкурсу» только «верные» модели.

2. В приложении к геосреде его использование нередко означает отождествление структуры геологической среды с особенностями движения другой (геофизической) среды, что, как подчеркивалось выше, не подтверждается наблюдениями.

3. Часто поведение физических величин (и, следовательно, поведение системы), которое прогнозируется априори для некоторого интервала масштабных оценок типа (1), не меняется кардинально и при существенном нарушении этих оценок. Так, в механике хрупкого разрушения предполагалось, что упругая сингулярная асимптотика напряжений, $\sim k r^{-1 / 2}$, где $k$ - коэффициент интенсивности напряжений, для реальных тел остается справедливой на расстояниях $r$ от вершины трещины, если $d_{p}<<r<<L$, где $d_{p}-$ размер пластической зоны у вершины трещины. Практика показывает, однако, что такое поведение напряжений сохраняется и тогда, когда $d_{p}$ достигает размеров порядка десятков процентов от длины трещины L [Parton, 1990]. При этом энергетическая концепция критического коэффициента, $k=k_{c}$, сохраняется, быть может, при некотором фиктивном изменении $L$. Отмеченное обстоятельство позволяет не видоизменять эту концепцию при относительно развитых пластических зонах [Mukhamediev et al., 1976].

Итак, неприятие континуального подхода к моделированию движения дискретной по строению среды, которое базируется на «вкусовых» предпочтениях или формальных априорных оценках, не может отвергнуть возможности его применимости на уровне количественных моделей. В разделе 2.3 мы представим убедительный пример из физики пространства-времени, поддерживающий наше мнение. Возникает вопрос: какие требования, кроме применимости, следует предъявить модели движения геосреды? Здесь наша точка зрения, в отличие от некоторых других аспектов, совпадает с мнением большинства - в модели должны выполняться законы сохранения, отражающие законы природы. Невыполнение этих законов не позволяет считать модель физической. Ее предсказания становятся ненадежными, или количественные соотношения в ней превращаются в набор эмпирических правил, лишенных прогностических свойств.

\section{3. ПРИМЕРЫ КОНТИНУАЛЬНОГО ПОДХОДА К ОПИСАНИЮ ДИСКРЕТНОЙ И ДИСКРЕТНО-ИЕРАРХИЧЕСКОЙ СРЕД}

Континуальные модели привлекательны из-за возможности формулировки задачи в терминах гладких (или кусочно-гладких) полевых функций, удовлетворяющих дифференциальным уравнени- ям в частных производных. Методы решения многих таких уравнений хорошо разработаны. Согласно отмеченному, принципиально важным остается вопрос о применимости этих моделей для выявления тех или иных свойств дискретных систем или систем, которые интерпретируются как дискретные. Отдельные ответы на этот вопрос получены в разных областях знаний.

Например, дискретность строения Вселенной и иерархичность этого строения (планеты $\rightarrow$ звезды и планетные системы $\rightarrow$ галактики $\rightarrow$ скопления галактик) выражены не менее убедительно, чем в случае геосреды. Более того, наблюдаемые отдельности во Вселенной не контактируют друг с другом, осуществляя, согласно традиционным воззрениям, взаимное силовое воздействие на расстоянии. Если следовать мнению противников моделей сплошности, то было бы ошибкой изучать эволюцию и свойства Вселенной на основе континуального подхода. Тем не менее полевые уравнения общей теории относительности (ОТО) Эйнштейна, которые в каждой точке среды связывают тензор энергии-импульса с метрическими свойствами пространства-времени, хорошо справляются с этой задачей. Основные постулаты ОТО и некоторые решения ее уравнений недавно еще раз выдержали экспериментальную проверку. Во-первых, космической миссией Gravity Probe B обнаружен эффект искривления пространства-времени, количественно совпадающий с предсказаниями ОТО [Everitt et $a l ., 2011]$, и, во-вторых, решения линеаризованной системы уравнений ОТО подтверждены обнаружением гравитационных волн в лабораторных условиях [Abbott et al., 2016]. С момента зарождения ОТО стали находиться точные решения уравнений, к которым относятся, например, статическое сферически-симметричное решение Шварцшильда (1916), описывающее черную дыру [Kruskal, 1960], и решение для вращающейся черной дыры [Kerr, 1963]. На основе ОТО строятся современные стационарные и нестационарные космологические модели Вселенной. Таким образом, пример ОТО убеждает нас в том, что континуальная модель может быть вполне применима и работоспособна при изучении среды с наблюдающимися свойствами дискретности, иерархичности и дальнодействия. В нашей классификации ОТО не относится к классу «верных» моделей и уж тем более не подчиняется оценкам типа (1). Тем не менее в зоне своей ответственности она адекватно описывает многие глобальные и локальные свойства среды, имеет предсказательную силу и подсказывает новые идеи.

Возвращаясь к земным проблемам, отметим, что и здесь существуют и успешно справляются с возникающими проблемами континуальные модели, 
правда, намного более частные, если сравнивать с ОТО. Во многих моделях дискретность, рассматриваемая как микроструктура, гомогенизирована, а ее влияние учитывается опосредованно с помощью некоторых макроскопических параметров и/или функций. При этом может возникнуть необходимость в придании макроскопическим деформациям и напряжениям особого смысла, отличного от того, который они имеют в среде без микроструктуры. Так, уже много десятилетий при исследовании фильтрации флюидов в трещиноватых и трещиновато-пористых массивах горных пород используют модели сплошной среды, например модель двойной пористости. В этих случаях макроскопическим напряжениям, связанным с деформациями твердого скелета среды, следует придать смысл эффективных, тем или иным способом учитывая давление флюида, а проницаемость пород рассматривать как анизотропную, с ориентацией главных осей тензора проницаемости, зависящей от ориентации систем проводящих трещин. В итоге массив, фактически имеющий дискретное строение, моделируется сплошной двухфазной средой. Почему такая ситуация не вызывает возражения или удивления у противников континуального подхода? Ответ заключается в том, что никого не интересуют потоки флюида на масштабе отдельной поры или трещины.

Сказанного выше достаточно для того, чтобы не отвергать огульно концепцию сплошности, а попробовать разобраться в ее применимости к моделированию движения геосреды. С целью более предметного обсуждения проблемы следует коротко очертить современное состояние механики твердого деформируемого тела (ТДТ), модель которого ниже в основном и рассматривается в качестве континуальной модели геосреды, а также затронуть особенности использования модели ТДТ в задачах геологии и геофизики.

\section{3. ЭЛЕМЕНТЫ КЛАССИЧЕСКОЙ МОДЕЛИ ТВЕРДОГО ДЕФОРМИРУЕМОГО ТЕЛА}

Рассмотрим очень кратко некоторые стандартные положения континуального подхода в свете их приложений к проблемам геосреды. Многие из приводимых положений и соотношений в том или ином виде и с точностью до обозначений можно найти в современных курсах сплошной среды (например [Truesdell, 1972]). Мы применяем частично дополненную систему обозначений Гиббса, совпадающую с использованной в монографии [Mukhamediev, 1990]. Подробное изложение тензорного исчисления в этих обозначениях приведено в пособии [Ryzhak, 2011].

\section{1. КИНЕМАТИКА}

В механике ТДТ при отсчетном описании деформации тела $B$ выбирается некоторая его конфигурация к, которая принимается за отсчетную (или начальную в момент $t=0$ ). В конфигурации к частицам тела относят в соответствие радиус-векторы их положения $\mathbf{x}$. Для простоты саму частицу 3 будем также обозначать символом х. Эту частицу называют по-разному: телом-точкой, материальной точкой, лагранжевой частицей, материальным элементом. При построении модели структурированных или сильно неоднородных материалов выбор размера тела-точки, не совпадающего, как правило, с размерами реальных структурных элементов (зерен, блоков и т.д.), часто представляет собой нетривиальную проблему. Реальные неоднородности внутри тела-точки гомогенизируются, модельная среда приобретает смысл эффективной, а ее частица $\mathbf{x}^{\text {eff }}$ - смысл репрезентативного (макро)объема, рассматриваемого как элементарный в пространственном масштабе эффективной среды 4 . Задача механики ТДТ полностью решена, если при заданных определяющих соотношениях материала тела $B$ в каждый момент времени $t$ известно положение $\mathbf{r}$ каждой частицы тела $B$, т.е. задана дифференцируемая (или кусочно-дифференцируемая) вектор-функция, осуществляющая взаимно-однозначное отображение $\mathbf{x} \rightarrow \mathbf{r}$,

$$
\mathbf{r}=\mathbf{r}(\mathbf{x}, t), \forall \mathbf{x} \in B
$$

Для упругого тела достаточно знать пространственное положение $\mathbf{r}$ частиц $\mathbf{x}$ не во все моменты времени, а только в актуальной (текущей) конфигурации. Потеря непрерывности отображением (2) на множестве меры 0 в $B$ означает наличие явным образом вводимых в модель ТДТ разрывов - трещин, контактных границ раздела с проскальзыванием и т.д. Возможность использования кусочногладкого поточечного отображения (2) есть важнейший признак (достаточный, но необязательно необходимый) континуального подхода к относительному описанию движения тела $B$. Ниже мы в основном ограничимся случаем, когда отображение (2) существует, хотя это и оставляет вне поля зрения широкий класс континуальных моделей, в которых условие совместности деформаций не выполняется и которые потенциально применимы к исследованию важных процессов в геосреде.

\footnotetext{
3 В континуальных моделях термин «частица» относится к математическому объекту в отличие от дискретных моделей, где этот термин означает физический объект.

${ }^{4} \mathrm{C}$ вопросами гомогенизации сред со структурой, построения эффективной среды и выбора $\mathbf{x}_{\text {eff }}$ можно ознакомиться, например, по работам [Nemat-Nasser, Hori, 1993; Bayuk, 2013].
} 
Кинематика среды в малой окрестности частицы $\mathbf{x}$ определяется тем, как линейный материальный элемент $d \mathbf{x}$ отображается в пространственный элемент $d \mathbf{r}(\mathbf{x}, t, d \mathbf{x})$. В первом приближении отображение $d \mathbf{x} \rightarrow d \mathbf{r}$ линейное, поэтому оно осуществляется тензором второго ранга $\mathbf{F}, d \mathbf{r}=d \mathbf{x} \cdot \mathbf{F}$, где символ • означает скалярное умножение. Отсюда следует, что

$$
\mathbf{F}(\mathbf{x}, t)=\nabla_{\mathbf{K}} \otimes \mathbf{r}(\mathbf{x}, t)(\operatorname{det} \mathbf{F}>0),
$$

где наличие индекса к у символа градиента $\nabla$ указывает на дифференцирование по переменным $\mathbf{x}$ отсчетной конфигурации $(\otimes-$ символ тензорного умножения). Тензор макроскопической дисторсии $\mathbf{F}$ приписывается самой частице $\mathbf{x}$, он несимметричен, и в нем одновременно содержится информация и о деформации, и о вращении малой окрестности точки $\mathbf{X}$, что позволяет выразить через $\mathbf{F}$ различные локальные деформационные и ротацион-

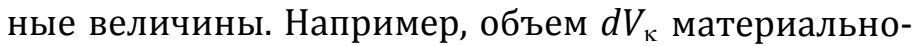
го элемента и ориентированный материальный элемент поверхности $d \mathbf{s}_{\kappa}=\mathbf{n}_{\kappa} d s_{\kappa}$ ( $\mathbf{n}_{\kappa}-$ вектор единичной нормали, $d s_{\kappa}-$ площадь), заданные в конфигурации к, отображаются в их образы $d V$ и $d \mathbf{s}$ в актуальной конфигурации по правилам:

$$
d V=(\operatorname{det} \mathbf{F}) d V_{\kappa}, d \mathbf{s}=(\operatorname{det} \mathbf{F}) d \mathbf{s}_{\mathrm{\kappa}} \cdot\left(\mathbf{F}^{-1}\right)^{T},
$$

где верхние индексы ${ }^{-1}$ и $^{T}$ обозначают обратный и транспонированный тензоры, det - детерминант. Материал, напряжения в котором при фиксированной конфигурации к определяются только историей изменения $\mathbf{F}$, в рациональной механике называется простым. Именно модель такого материала рассматривается в разделе 3 .

При линеаризации кинематических соотношений в окрестности некоторого состояния, т.е. при преобразовании этих соотношений к привычному для многих случаю малых деформаций, отсчетная конфигурация к принимается в качестве актуальной, тензор F превращается в единичный тензор I, а его приращение $\delta \mathbf{F}$ представляется в виде:

$$
\begin{aligned}
& \delta \mathbf{F}(\mathbf{x}, t)=\nabla \otimes \delta \mathbf{u}(\mathbf{x}, t)=\delta \mathbf{E}+\delta \mathbf{W}, \\
& \delta \mathbf{E}=\frac{1}{2}\left(\delta \mathbf{F}+\delta \mathbf{F}^{T}\right), \quad \delta \mathbf{W}=\frac{1}{2}\left(\delta \mathbf{F}-\delta \mathbf{F}^{T}\right) .
\end{aligned}
$$

Здесь $\delta \mathbf{u}$ - вектор малых макросмещений точек $\mathbf{x}$, $\delta \mathbf{E}$ и $\delta \mathbf{W}$ - соответственно, симметричная деформационная и антисимметричная ротационная составляющие инкрементальной дисторсии, причем $\delta \mathbf{W}=\delta \mathbf{W} \times \mathbf{I}$, где $\delta \mathbf{w}-$ вектор малого макроповорота, $\times$ - символ векторного умножения. 0 кинематике реальной геосреды часто приходится судить по раз-

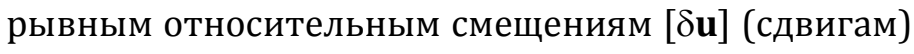

на площадках $d \mathbf{s}=\mathbf{n} d s$. В макрообъеме $\mathbf{r}$ eff= $\mathbf{r}\left(\mathbf{x}^{e f f}, t\right)^{5} \mathrm{c}$ объемом $d V$ такой сдвиг порождает приращение микродисторсии $d \mathbf{s} \otimes[\delta \mathbf{u}] / d V$, что в результате $N$ подвижек приводит к приращению макродисторсии:

$$
\delta \mathbf{F}=\frac{1}{d V} \sum_{i=1}^{N} d \mathbf{s}_{(i)} \otimes[\delta \mathbf{u}]_{(i)},
$$

которое, согласно (5), можно разложить на сумму приращений деформации и вращения. При использовании данных о фокальных механизмах землетрясений неясно, какая из двух плоскостей (с нор-

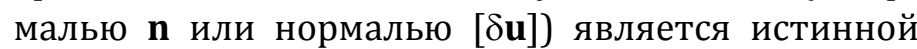
площадкой подвижки. В этом случае можно рассчитать симметричный тензор $\delta \mathbf{E}$, но не $\delta \mathbf{F}$, что означает потерю информации о повороте $\delta \mathbf{w}$ макрообъема $\mathbf{x}$ как целого. Представление макродисторсии в виде (6) является важнейшим шагом на пути континуализации движения геосреды.

\section{2. НАПРЯЖЕНИЯ И ЗАКОНЫ СОХРАНЕНИЯ ДЛЯ КОНТИНУУМА КОШИ}

Коши (1789-1857) при построении своей модели среды ввел явное предположение, получившее название постулата Коши. Согласно этому постулату, контактное воздействие частей тела, разделенных мысленно выделенной внутри тела поверхностью, в малой окрестности каждой точки поверхности описывается силой $d \mathbf{f}$, которая зависит лишь от касательной к поверхности (а не, скажем, от ее кривизны). Математически это представляется как $d \mathbf{f}=d \mathbf{f}\left(d \mathbf{s}_{\kappa}, \mathbf{x}\right)=d \mathbf{f}(d \mathbf{s}, \mathbf{r}(\mathbf{x}, t))$, где $d \mathbf{s}_{\kappa}$ и $d \mathbf{s}-$ ориентированные касательные площадки в отсчетной и актуальной конфигурациях. При смене ориентации площадки на противоположную вектор $d \mathbf{f}$ также меняет свою ориентацию на противоположную, $d \mathbf{f} \rightarrow-d \mathbf{f}$. Далее, согласно теореме Коши, из непрерывности $d \mathbf{f}$, как функции от $d \mathbf{s}_{\kappa}$ или $d \mathbf{s}$, следует линейность этой функции, т.е. существуют такие тензоры второго ранга $\mathbf{T}_{\kappa}$ и $\mathbf{T}$, что

$$
d \mathbf{f}=d \mathbf{s} \cdot \mathbf{T}=d \mathbf{s}_{\mathrm{\kappa}} \cdot \mathbf{T}_{\mathrm{\kappa}} .
$$

Тензоры $\mathbf{T}$ и $\mathbf{T}_{\kappa}$ называются, соответственно, тензорами напряжений Коши и Пиолы (1794-1850). Тензор Пиолы известен также как тензор напряжений Пиолы-Кирхгофа 2-го рода или тензор номинальных напряжений. Так как способ введения тензоров $\mathbf{T}$ и $\mathbf{T}_{\kappa}$ опирается на представление о силе, действующей на площадке, мы называем эти тензоры силовыми, чтобы отличать их, например,

\footnotetext{
5 В отличие от $\mathbf{x}^{e f f}$ макрообъем $\mathbf{r}$ eff уже не материальный, а пространственный.
} 
от тензоров моментных напряжений. Тензор Т традиционно используется при пространственном описании движения, тензор $\mathbf{T}_{\kappa}-$ при отсчетном.

Согласно второму соотношению в (4), между $\mathbf{T}$ и $\mathbf{T}_{\kappa}$ существует связь:

$$
\mathbf{T}_{\mathrm{K}}=(\operatorname{det} \mathbf{F})\left(\mathbf{F}^{-1}\right)^{T} \cdot \mathbf{T} .
$$

Отметим, что при создании своей модели Коши, помимо отмеченных предположений, неявно вводил запреты на существование моментных напряжений (couple stresses), а также на дополнительные степени свободы элемента $\mathbf{x}$. В результате тензор $\mathbf{T}$ (или $\mathbf{T}_{\kappa}$ ) явился единственным объектом, ответственным за динамическое состояние сплошной среды. Такую модель простого материала называют континуумом Коши.

В любой корректно построенной модели среды, в том числе континуальной, должны выполняться законы сохранения. Под законами сохранения какой-либо величины мы понимаем не только неизменность этой величины в замкнутой системе, вовлеченной в некоторые специальные типы движения, но иногда и балансовые соотношения, которым должна удовлетворять эта величина в случае наличия ее источников и стоков в системе, а также обмена ею с окружающей средой. В этом широком понимании законы сохранения импульса и момента импульса 6 формируют, фактически, уравнения движения системы.

Из закона сохранения массы $\rho d V=\rho_{\kappa} d V_{\kappa}$ и первого соотношения в (4) связь между плотностью $\rho_{\kappa}$ частицы $\mathbf{X}$ в конфигурации к и ее актуальной плотностью $\rho$ имеет вид:

$$
\rho_{\kappa}=\rho(\operatorname{det} \mathbf{F})
$$

Если пренебречь инерционными членами, то закон сохранения импульса влечет за собой условия равновесия, которые в терминах тензоров $\mathbf{T}$ и $\mathbf{T}_{\kappa}$ записываются как

$$
\begin{aligned}
& \nabla \cdot \mathbf{T}(\mathbf{r}(\mathbf{x}, t))+\rho(\mathbf{r}(\mathbf{x}, t)) \mathbf{g}=0 \\
& \nabla_{\boldsymbol{\kappa}} \cdot \mathbf{T}_{\kappa}(\mathbf{x}, t)+\rho_{\kappa}(\mathbf{x}) \mathbf{g}=0,
\end{aligned}
$$

где $\mathbf{g}$ - ускорение свободного падения, $\nabla \cdot$ и $\nabla_{\kappa} \cdot-$ символы дивергенции при дифференцировании, соответственно, по $\mathbf{r}$ и $\mathbf{x}$. Закон сохранения момента импульса при выполнении соотношений (9) требует от $\mathbf{T}$ и $\mathbf{T}_{\kappa}$ следующих условий симметрии:

\footnotetext{
6 Эти законы, впервые совместно и в строгой математической форме введенные Эйлером (1707-1783) в 1766 г. [Mikhailov, 2007], получили название 1-го и 2-го законов динамики Эйлеpa.
}

$$
\mathbf{T}=\mathbf{T}^{T}, \quad \mathbf{F}^{T} \cdot \mathbf{T}_{\kappa}=\mathbf{T}_{\kappa}^{T} \cdot \mathbf{F} .
$$

Тензор $\mathbf{T}_{\kappa}$ несимметричен. Причина этого кроется не в физике, а в геометрии: линейное преобразование $d \mathbf{s}_{\kappa} \rightarrow d \mathbf{s}$ осуществляется несимметричным тензором. Тензор $\mathbf{T}_{\kappa}$ становится симметричным лишь тогда, когда в качестве отсчетной используется актуальная конфигурация (см. раздел 3.3).

\section{3. ОПРЕДЕЛЯЮЩИЕ СООТНОШЕНИЯ В КОНТИНУУМЕ КОШИ}

Простые упругие материалы в рамках механической теории отличаются от других тем, что в каждой частице $\mathbf{x}$ обратимым образом преобразуют всю накопленную дисторсию в упругую энергию. Друг от друга же простые упругие материалы отличаются способом преобразования дисторсии в энергию, т.е. функцией $e_{\kappa}(\mathbf{F}, \mathbf{x})$ зависимости плотности упругой энергии $e_{\text {к }}$ (на единицу объема в конфигурации к) от дисторсии $\mathbf{F}$, введенной соотношением (3). Функция $e_{\kappa}(\mathbf{F}, \mathbf{x})$ является упругим потенциалом, $\mathbf{T}_{\kappa}=\partial e_{\kappa} / \partial \mathbf{F}$, что влечет за собой определяющее соотношение упругости $\mathbf{T}_{\kappa}=\mathbf{T}_{\kappa}(\mathbf{F}, \mathbf{x})$. Для однородного материала зависимость от $\mathbf{x}$ пропадает. Дальнейшая конкретизация определяющего соотношения достигается на основе принципа его независимости от выбранной системы отсчета.

Линеаризуем предъявленное определяющее соотношение упругости в окрестности некоторого актуального состояния с ненулевым тензором начальных напряжений $\mathbf{T}_{0}$. Как и в разделе 3.1 , актуальная конфигурация принимается за отсчетную, т.е. $\mathbf{F}=\mathbf{I}$. Тогда $\mathbf{T}_{\kappa}=\mathbf{T}=\mathbf{T}_{0}$, но и в этом случае запись приращения $\delta \mathbf{T}_{\text {к }}$ через приращения кинематических макрохарактеристик осуществляется иначе, чем запись выражения для $\delta \mathbf{T}$. Получаем

$$
\begin{aligned}
& \delta \mathbf{T}=\underline{\mathbf{L}}: \delta \mathbf{E}+\mathbf{T}_{0} \cdot \delta \mathbf{W}-\delta \mathbf{W} \cdot \mathbf{T}_{0} \\
& \delta \mathbf{T}_{\kappa}=\underline{\mathbf{L}}: \delta \mathbf{E}+\mathbf{T}_{0}(\mathbf{I}: \delta \mathbf{E})-\delta \mathbf{E} \cdot \mathbf{T}_{0}+\mathbf{T}_{0} \cdot \delta \mathbf{W},
\end{aligned}
$$

где имеющий определенные свойства симметрии тензор 4-го ранга $\underline{\mathbf{L}}=\underline{\mathbf{L}}\left(\mathbf{T}_{0}, \mathbf{x}\right)$ является тензором упругих модулей, которыми материал обладает при напряжениях $\mathbf{T}=\mathbf{T}_{0}$, : - символ двойного скалярного умножения, приращения $\delta \mathbf{E}$ и $\delta \mathbf{W}$ определены в (5). Лишь при отсутствии начальных напряжений приращения тензоров напряжений Коши и Пиолы неразличимы.

В большинстве геолого-геофизических работ при исследовании процессов (в частности, волновых) в упругой геосреде без всяких объяснений и оценок влиянием начальных напряжений пренебрегают. Неявное обнуление реально существующего в геосреде начального напряжения приводит иногда к следующим ошибкам. 
1. Вместо тензора $\underline{\mathbf{L}}\left(\mathbf{T}_{0}\right)$ используется тензор $\underline{\mathbf{L}}(0)$, т.е. в качестве упругих модулей выбираются их значения при нулевых напряжениях.

2. При подсчете изменения напряжения, сопровождающего заданную дисторсию $\delta \mathbf{F}$, не учитывается тот факт, что вращение недеформируемой частицы $\mathbf{x}$ не вызывает изменение тензора т в системе отсчета, жестко связанной с х. На самом деле, заданному тензору $\underline{\mathbf{L}}: \delta \mathbf{E}$ соответствует объективное (яуманново) приращение напряжений $\delta^{\nabla} \mathbf{T}=$ $=\delta \mathbf{T}+\delta \mathbf{W} \cdot \mathbf{T}_{0}-\mathbf{T}_{0} \cdot \delta \mathbf{W}$.

3. Деформации отсчитываются от разгруженного состояния массива, в котором массив горной породы никогда не пребывал, и этим фиктивным деформациям по законам упругости ставятся в соответствие напряжения, которые трактуются как реальные.

Отмеченные ошибки, типичные для ряда геолого-геофизических работ, могут привести к качественно неверным результатам, например, при расчетах напряженного состояния и при исследовании распространения волн. Более того, не учитывая начальные напряжения (или не умея их учитывать), некоторые авторы этот недостаток приписывают не себе, а континуальной модели, заявляя, что влияние начальных напряжений в рамках модели сплошной среды учтено быть не может (см. раздел 6.1).

Модель упругой материальной частицы х предписывает ей абсолютную память относительно любого ее отсчетного состояния ${ }^{7}$ и полный перевод работы в упругую энергию при деформировании х. Рассмотрим противоположную идеализацию: частица $\mathbf{x}$ мгновенно забывает свое предыдущее состояние (а именно предыдущую скорость и напряжение) и полностью рассеивает работу в каждый момент своего движения. Геосреду, составленную из таких частиц, мы назвали идеальнодиссипативной [Mukhamediev, 1990]. При описании этой среды (в силу ее мгновенно затухающей памяти) естественно пользоваться не приращениями величин, а скоростями их изменения, и перейти от отсчетного описания движения к пространственному. Относя приращения $\delta \mathbf{E}, \delta \mathbf{W}$ к соответствующему интервалу времени $t$, из (3), (5) имеем:

$$
\begin{aligned}
& \dot{\mathbf{F}}=\nabla \otimes \mathbf{v}, \dot{\mathbf{E}}=\frac{1}{2}\left(\nabla \otimes \mathbf{v}+\nabla \otimes \mathbf{v}^{T}\right), \\
& \dot{\mathbf{W}}=\frac{1}{2}\left(\nabla \otimes \mathbf{v}-\nabla \otimes \mathbf{v}^{T}\right),
\end{aligned}
$$

где $\mathbf{v}(\mathbf{r}, t)$ - скорость той частицы $\mathbf{x}$, которая в данный момент $t$ оказалась в пространственной точке $\mathbf{r}, \dot{\mathbf{F}}$ - градиент скорости, $\dot{\mathbf{E}}$ - тензор скорости деформации, $\mathbf{W}-$ спин. Аналогично тому, как упругую

\footnotetext{
7 При этом частица, из-за ненадобности, не помнит свой путь из отсчетного состояния.
}

среду характеризуют упругим потенциалом, однородный идеально-диссипативный материал можно характеризовать диссипативной функцией (или функцией рассеяния) $\Phi(\dot{\mathbf{E}})$, т.е. зависимостью диссипации работы внутренних сил $\mathbf{T}: \dot{\mathbf{E}}$, отнесенной к единичному пространственному объему, от скорости деформации. В класс идеально-диссипативных сред, например, входят несжимаемые $(\mathbf{I}: \dot{\mathbf{E}}=0)$ изотропные вязкие жидкости, определяющие соотношения для которых имеют вид:

$$
\mathbf{T}^{d e v}=F\left(J_{2}(\dot{\mathbf{E}}), J_{3}(\dot{\mathbf{E}})\right) \dot{\mathbf{E}},
$$

где $\mathbf{T}^{d e v}$ - девиатор напряжений Коши, $F$ - заданная функция второго и третьего инвариантов $J_{2}$ и $J_{3}$ тензора $\dot{\mathbf{E}}$, причем $F>0$ при любом $\dot{\mathbf{E}}$.

Широкий подкласс идеально-диссипативных материалов составляют идеальные пластические среды. С использованием экстремального принципа теорию таких сред можно строить как на основе функции $\Phi(\dot{\mathbf{E}})$, так и на основе пластического потенциала, роль которого играет поверхность текучести $\psi:\left\{\psi\left(\mathbf{T}^{d e v}\right)=\right.$ const $\}$ такая, что $\mathbf{T}^{d e v} \in \psi$ в процессе деформирования [Ivlev, 1966]. Для однородного изотропного несжимаемого материала, опираясь на принцип максимума Мизеса, получаем [Mukhamediev, 1993]:

$$
\begin{aligned}
& \dot{\mathbf{E}}=\lambda \frac{\partial \psi}{\partial I_{3}}\left(\mathbf{T}^{d e v} \cdot \mathbf{T}^{d e v}+\frac{2}{3} I_{2} \mathbf{I}\right)-\lambda \frac{\partial \psi}{\partial I_{2}} \mathbf{T}^{d e v} \\
& \left(\mathbf{T}^{d e v} \in \Psi, \mathbf{I}: \dot{\mathbf{E}}=0\right),
\end{aligned}
$$

где $\lambda>0$ - произвольная константа, $I_{2}$ и $I_{3}-$ второй и третий инварианты тензора $\mathbf{T}^{d e v}$. В пластической среде, в отличие от вязкой, напряжения принадлежат поверхности текучести и скорость деформаций виртуальна в том смысле, что физическое время $t$ можно заменить на любую монотонно возрастающую с ростом $t$ функцию.

\section{4. УЧЕТ МИКРОСТРУКТУРЫ И ОБОБЩЕНИЯ КОНТИНУУМА КОШИ}

Существует большое разнообразие сред, в поведении которых особенности строения играют важную роль. Например, для геосреды наблюдается масштабный эффект зависимости макроскопических физических свойств от пространственных размеров и длительности протекания процесса. Второе из отмеченных явлений можно учесть в рамках континуума Коши, рассмотрев, в частности, локальные вязкоупругие определяющие соотношения. Однако в тех моделях геосреды, где ставится задача учесть пространственный эффект, приходится выходить за рамки континуума Коши, так 
как определяющие соотношения последнего не содержат параметра размерности длины. В механике предлагалось множество подходов к учету микроструктуры, отраженных в столь огромном количестве литературных источников, что сколько-нибудь обстоятельный обзор их представляется нереальным. В настоящем разделе мы ограничимся некоторыми замечаниями.

\section{1. СПОСОБЫ ПРЯМОЙ КОНТИНУАЛИЗАЦИИ ДИСКРЕТНОЙ СРЕДЫ}

В атомистических теориях и методах молекулярной динамики подробно учитывается исходная дискретность среды. Численное моделирование на основе методов дискретных элементов поставляет информацию о траекториях частиц и силах, действующих на отдельные частицы [Zhu et al., 2008; и др.]. Приложение этих методов к анализу геологических структур обсуждается в [Gray et al., 2014]. В России наиболее активным сторонником подхода к моделированию среды на основе явного учета ее дискретности был В.Н. Страхов [Strakhov, 2007]. Некоторые сведения об ограничениях на вычислительные возможности прямых методов и о современных способах осреднения в дискретных средах подобного типа можно найти в [Davydov et al., 2014; Li, Urata, 2016].

Обычный вариант континуализации динамических свойств атомистической модели основывается на теореме вириала Клаузиуса, которая приводится в пособиях по теоретической механике. В рамках этой модели для репрезентативного объема $V \subseteq V_{0}$, где $V_{0}$ - объем, который занимает весь ансамбль частиц, совершающих периодические или финитные движения, строится тензор вириальных напряжений, $\mathbf{T}^{v i r}=\mathbf{T}^{k i n}+\mathbf{T} f$, где

$$
\begin{aligned}
& \mathbf{T}^{\text {kin }}=-\frac{1}{V} \sum_{\alpha \in V} m_{\alpha} \mathbf{v}_{\alpha} \otimes \mathbf{v}_{\alpha} \\
& \mathbf{T}^{f}=\frac{1}{2 V} \sum_{\alpha \in V} \sum_{\beta \in V_{0}}\left(\mathbf{r}_{\beta}-\mathbf{r}_{\alpha}\right) \otimes \mathbf{f}_{\alpha \beta} .
\end{aligned}
$$

Здесь $\mathbf{v}_{\alpha}-$ скорость $\alpha$-й частицы, которая обладает массой $m_{\alpha}$ и центр которой в актуальном состоянии расположен в точке $\mathbf{r}_{\alpha}, \mathbf{f}_{\alpha \beta}-$ сила, с которой частица $\beta$ воздействует на частицу $\alpha\left(\mathbf{f}_{\alpha \beta}=-\mathbf{f}_{\beta \alpha}\right)$. Тензор кинетических напряжений, $\mathbf{T}^{k i n}$, связан с переносом импульса движущимися частицами, а тензор силовых напряжений т $f$ отражает силы дальнодействия. Тензор $\mathbf{T} f$ (а следовательно, и $\mathbf{T}^{v i r}$ ) симметричен, так как векторы $\mathbf{f}_{\alpha \beta}$ и $\mathbf{r}_{\beta}-\mathbf{r}_{\alpha}$ параллельны. В литературе дебатируется вопрос о том, какой из тензоров - $\mathbf{T}^{f}$ или $\mathbf{T}^{v i r}$ - можно считать эквивалентом тензора T в континууме Коши [Zhou, 2003; Subramaniyan, Sun, 2008]. Интересно отметить, что в силу эффективной двухмерности актуальной кон- фигурации Солнечной системы и спиральных галактик тензор Tvir для них будет почти плоским. Возможность введения континуальной характеристики Tvir для такой системы, как Солнце с планетами, может показаться кощунственной для противников континуального подхода.

Вышеприведенные идеи можно перенести на гранулированную среду, описание которой зависит от масштаба рассмотрения. На микромасштабе гранулированный материал представляется совокупностью индивидуальных зерен; описание его дискретного поведения проводится в терминах перемещения частиц и контактных сил взаимодействия между ними без введения напряжений. Введение тензора напряжений на макромасштабе при явном учете микроструктуры проводится разными способами, но, в общем, представление тензора напряжений Tgran вполне аналогично представлению тензора $\mathbf{T}^{v i r}$ с той разницей, что символами $\mathbf{f}_{\alpha \beta}$ теперь обозначаются силы контактного взаимодействия между смежными зернами $\alpha$ и $\beta$, скорости $\mathbf{v}_{\alpha}$ являются отклонениями от средних (например [Goldhirsch, Goldenberg, 2005]). При контактах без трения тензор Тgran, очевидно, симметричен.

Упомянем еще перидинамическую модель, претендующую на обобщение механики континуума Коши, в котором внутренние силы взаимодействия вводятся только для контактирующих частиц. В перидинамической модели, как и в атомистической, вводятся нелокальные силы взаимодействия между отдаленными частицами, однако взаимодействие ограничено некоторым предельным расстоянием $r$ [Silling, Lehoucq, 2010]. Эта модель сейчас позиционируется как новая, но фактически идея о предельном расстоянии взаимодействия $r$ (называемом радиусом сферы молекулярного действия) предлагалась как самим Коши (еще в 1827 г.), так и Пиолой [Dell'Isola et al., 2014], а также другими исследователями [Love, 1927]. В перидинамической модели делается попытка описать сплошную среду с дефектами (типа трещин и включений) в рамках единого подхода. Перидинамический тензор напряжений Трer аналогичен тензору напряжений Пиолы $\mathbf{T}_{\text {к }}$ в континууме Коши в том смысле, что он, как и тензор $\mathbf{T}_{\kappa}$, отображает элементарные площадки $d \mathbf{s}_{\kappa}$ в отсчетной конфигурации тела в векторы приложенных к $d \mathbf{s}_{\kappa}$ сил. Разница заключается в том, что в перидинамической модели силы не локальны. Предельный переход в континуум Коши происходит при $r \rightarrow 0$ [Silling, Lehoucq, 2008].

\section{2. ОБ ОБОБЩЕНИЯХ КОНТИНУУМА КОШИ}

Как ни странно, модели среды, в которых учитываются распределенные моменты и которые 
сейчас рассматриваются как обобщения континуума Коши, возникли задолго до рождения Коши. Еще в 1694 г. Я. Бернулли (1655-1705), решая задачу об изгибе упругой балки и выдвигая гипотезу плоских сечений, получил уравнение, которое можно записать как $M=C \mathrm{~K}$, где $M$ - изгибающий момент, $\mathrm{K}$ - кривизна изогнутой оси балки, а коэффициент пропорциональности $C$ является произведением модуля Юнга $E$ и момента инерции сечения [Zhilin, 2006]. Это уравнение используется в сопромате до сих пор при определении прогибов и упругой линии балки в частных случаях изгиба. В 1771 г. Эйлер получил общие уравнения равновесия плоского изогнутого стержня, которые сохранились неизменными до наших дней [Zhilin, 2006]. Важно, что Эйлер, как и при выводе своего второго закона динамики, вводил момент как независимую самостоятельную величину, не сводящуюся к моменту силы. В уравнениях изгиба Эйлера-Бернулли динамические характеристики (растягивающее и перерезывающее усилия, а также изгибающий момент) удовлетворяют замкнутой системе дифференциальных уравнений, которую можно интерпретировать как систему уравнений 1D моментной теории упругости.

Отмеченные идеи были перенесены на 2D случай при построении теории изгиба пластин и оболочек, а затем и на 3D моментную (несимметричную) теорию упругости, излагаемую в настоящее время для случая малых деформаций в учебниках по теории упругости (например [Nowacki, 1970]). Здесь, в отличие от континуума Коши, за динамическое состояние сплошной среды ответственны два объекта - несимметричные тензоры силовых напряжений Т и моментных напряжений М. В квазистатическом случае в дополнение к уравнениям (10) возникает еще одно векторное уравнение равновесия относительно тензора М. Тензору Т ставится в соответствие несимметричный тензор деформации, а тензору $\mathbf{M}$ - так называемый тензор изгиба-кручения $\nabla \otimes \delta \mathbf{w}$, где $\delta \mathbf{w}-$ вектор макроповорота, упоминавшийся в разделе 3.1. При обобщении модели моментной среды элементарный объем $\mathbf{x}$ уже не рассматривается как примитивная тело-точка, он наделяется дополнительными степенями свободы. Обобщение на микрополярную среду8 в упругости достигается тем, что поворот частицы становится независимым от ее перемещения $\delta \mathbf{u}$. Таким образом, в упругом континууме Коссера тела-точки представляют собой абсолютно твердые тела, способные совершать независимые повороты и перемещения. Еще большая степень обоб-

\footnotetext{
8 Модель микрополярной среды называют континуумом Коссера по имени братьев Франсуа и Эжена Коссера, предложивших эту модель в 1909 г.
}

щения достигается для микроморфных сред, в моделях которых телу-точке приписывается собственная деформация. С развитием взглядов на механику усложненных моделей континуума и предлагавшимися количественными моделями можно ознакомиться по книгам [Eringen, 2002; Zhilin, 2003; Maugin, Metrikine, 2010].

Модели континуумов, отличных от континуума Коши, упомянуты нами выше не только с целью подчеркнуть потенциальные возможности идеи сплошности, но и потому, что в геолого-геофизической литературе эти модели, за редкими исключениями (например [Nikolaevsky, 1996; Garagash, Nikolaevskiy, 2009]), не используются. Более того, к таким моделям проявляется определенный скепсис, который выражается, в частности, в неприятии несимметричности тензоров силовых напряжений (см. раздел 6.1). При этом скептики упускают из вида, что моментные теории, во всяком случае одномерные, за более чем 200-летний период вполне доказали свою применимость хотя бы тем, что конструкции (здания, мосты и т.д.), спроектированные с их использованием, надежно служат человеку.

\section{3. О КОНТИНУАЛИЗАЦИИ ИСКУССТВЕННОЙ И ЕСТЕСТВЕННОЙ БЛОЧНОЙ СТРУКТУРЫ}

Как отмечалось выше, обсуждаемая врожденная дискретность строения среды не должна противопоставляться возможности использования континуального подхода к описанию ее движения. И действительно, этот подход (и, в частности, теория эффективной сплошной среды) в некоторых случаях, наряду с дискретным, стал активно развиваться при исследовании разного рода композитов, которые часто в упрощенном виде копируют элементы строения геосреды. В первую очередь это относится к моделям деформирования и разрушения каменной или кирпичной кладки, которые важны для строительной механики и проблем сохранности памятников архитектуры [Angelillo, 2014]. В кладке, с некоторыми поправками, относящимися к особенностям перевязки, кирпичи соответствуют блокам, межблоковое пространство, заполненное строительным раствором, - цементированным трещинам. Казалось бы, нет ничего проще, чем, точно скопировав геометрию всех элементов кладки и задав правила взаимодействия между блоками, численно исследовать все подробности напряжений и деформаций. Но часто (особенно для систем с большим числом элементов) вопросы глобальной устойчивости кладки и ее макроразрушения имеют больший приоритет, чем точное знание локальных подробностей, поэтому здесь, наряду с дискретным моделированием, стал развиваться континуальный подход. Он реализуется на основе разных способов 
осреднения [Lourenço et al., 2007] с использованием модели Коссера [Masiani, Trovalusci, 1996] или физической теории пластичности [Yuen, Kuang, 2013] и континуального разрушения [Pelà et al., 2011].

В области прикладных задач геомеханики в последнее время ощущается всплеск интереса к моделированию геосреды в связи с проблемами разработки трудноизвлекаемых запасов углеводородов. Финансово-затратные технологии гидроразрыва пластов призвали к жизни модели, в которых факт наличия врожденной дискретности, обусловленной поверхностями напластования и ортогональными к ним системами естественных трещин, не ставится под сомнение (в отличие от академических дискуссий, представленных в разделе 5) и учитывается явно [Yew, Weng, 2015]. Естественные поверхности раздела принципиальным образом влияют на развитие трещин гидроразрывов, которые, в свою очередь, даже на расстоянии вызывают сложные процессы деформации на этих поверхностях [Galybin, Mukhamediev, 2014; Cheng et al., 2015]. Дополнительно к этому внимание исследователей стала привлекать проблема так называемого стимулированного объема пород, который образуется при использовании технологий добычи сланцевых углеводородов, основанных на бурении горизонтальных скважин в продуктивном пласте и создании гидроразрыва с помощью закачки «скользкой» воды (т.е. раствора, вязкость которого понижена на порядок по сравнению с обычной) [Turcotte et al., 2014]. Стимулированный объем охватывает область разветвленных трещин гидроразрыва и/или активизированных естественных трещин, его развитие регистрируется микросейсмическими событиями [Maxwell et al., 2010] и теоретически моделируется как на основе континуального [Norris et al., 2015a], так и дискретного [Norris et al., 2015b] подхода.

\section{5. МОДЕЛИ ВОЗНИКНОВЕНИЯ БЛОЧНОЙ СТРУКТУРЫ ГЕОСРЕДЫ}

Важнейшим наблюдаемым признаком дискретного строения геосреды является наличие систем дизъюнктивных нарушений (трещин, разломов, деформационных полос, материальных границ раздела), которые при математическом моделировании представляются как поверхности нулевой или малой (по сравнению с линейным размером области исследования) толщины. На этих поверхностях терпят разрыв либо функция $\mathbf{r}(\mathbf{x}, t)$ в (2), либо некоторые компоненты тензора дисторсии $\mathbf{F}(\mathbf{x}, t)$ в (3). Помимо этого (и вследствие этого) на контактных поверхностях с трением преломляются траектории напряжений (ТГН), что подтверждается ла- бораторными экспериментами, а также данными бурения [Wu et al., 2007; Lin et al., 2013]. Законы преломления ТГН теоретически устанавливаются [Mukhamediev, 2014] на основе того, что скачок на поверхности контакта с нормалью $\mathbf{n}$ испытывает проекция $\widetilde{\mathbf{T}}=(\mathbf{I}-\mathbf{n} \otimes \mathbf{n}) \cdot \mathbf{T} \cdot(\mathbf{I}-\mathbf{n} \otimes \mathbf{n})$ на поверхность тензора Коши T, а не (псевдо)вектор напряжения $\mathbf{t}=\mathbf{n} \cdot \mathbf{T}$, который, в силу равновесия, непрерывен. Последнее означает непрерывность касательных и нормальных (к поверхности) напряжений. Это приходится подчеркивать особо, так как в геолого-геофизической литературе можно встретить ошибочные заявления по этому поводу.

\section{1. ПРЕДСТАВЛЕНИЯ О ПРИОБРЕТЕННОЙ ДИСКРЕТНОСТИ}

Проблема возникновения и развития дизъюнктивных нарушений в геосреде интересовала исследователей давно. Теория прочности не может описать развитие нарушения в массиве, находящемся в неоднородном напряженном состоянии, а теория трещин не в состоянии объяснить возникновение нарушения, так как для своего применения нуждается в уже заданных априорно макротрещинах [Kondaurov et al., 1987]. В работе [Rudnicki, Rice, 1975] в рамках континуальной теории пластичности была показана возможность образования слоев локализации пластических деформаций с одновременно происходящими относительно малыми деформациями упругой разгрузки в остальной части тела. После появления этой работы часть исследователей стала отождествлять именно слои локализации с зарождающимися макроскопическими нарушениями в сплошной среде. Появлению такой интерпретации способствовало и то, что в 70-х годах прошлого столетия возможность зарождения систем слоев локализации была продемонстрирована как экспериментально [Revuzhenko et al., 1974; Stoyanov, 1977], так и (с учетом стеснения деформации массива) теоретически [Nikitin, Ryzhak, 1977].

Не затрагивая гипотез образования отдельностей при остывании разогретого вещества (например, вещества древней протолитосферы [Sherman, 2015]), рассмотрим кратко, как отмеченные успехи 40-летней давности повлияли на развитие количественных моделей образования и эволюции блочной структуры геосреды. Не в последнюю очередь здесь, видимо, сказалось то обстоятельство, что у исследователей имелись навыки решения задач в рамках стандартной теории ТДТ и отсутствовали подобные навыки для блоковой модели. В результате получили развитие отмеченные во Введении представления, согласно которым образование структур разрушения, ограничивающих разномасштабные блоки, является механическим процессом, происходящим в изначально однородной сплош- 
ной горной породе, изотропной по прочностным свойствам. Например, как важнейший исходный тезис это представление декларируется в монографии [Makarov et al., 2007, p. 45] следующим образом: «Блочная структура присуща всем без исключения твердым деформируемым телам и средам, но не как изначально заданная, а как проявляемая ими в процессах деформирования и разрушения. Фактически блоки - это замороженные в кристаллической среде диссипативные структуры, сформировавшиеся в процессе деформации нагружаемой среды». Авторы, исповедующие подобное представление, в своей деятельности остаются в рамках модели сплошности и классических постановок краевых задач механики ТДТ, хотя при этом подчас злоупотребляют терминологией современной теории динамических систем, синергетики и неравновесной термодинамики. При моделировании горной породы упругопластическим (или упругохрупким) ТДТ они используют возможности, которые в рамках классических постановок задач открывает перед ними произвол в априорном выборе конкретного закона пластичности, критериев «разрушения», обратных связей и т.д. На основе этого авторы приходят к результатам, которые, на их взгляд, можно трактовать как образование блочной (и даже фрактальной) структуры. Общим в разных подходах остается одно: авторы подвергают деформации воображаемую литифицированную и изначально не структурированную сплошную горную породу. Для получения желаемого результата им приходится либо привлекать к рассмотрению большие напряжения, если постулируются прочностные свойства, соответствующие неповрежденным литифицированным породам, либо предполагать «деградацию» этих свойств в процессе деформации. С некоторыми оговорками отмеченные результаты можно трактовать как иллюстрацию широких возможностей континуального подхода, но в качестве объяснения образования дискретной структуры геосреды выдвигаемые представления вызывают возражения из-за их несоответствия наблюдениям.

\section{2. ПРЕДСТАВЛЕНИЯ О ВРОЖДЕННОЙ ДИСКРЕТНОСТИ}

Возражения обоснуем на примере возникновения систем внутрислойных трещин в осадочных породах - важнейшего геологического индикатора блочного строения, структура которого воспроизводится и на других масштабах. В обзоре [Pollard, Aydin, 1988] напоминается о том, что американский геолог Г.К. Джильберт в 1882 г. обнаружил дренажную систему, образованную двумя взаимно ортогональными системами трещин в осадках постгляциального периода. Существование трещин в столь молодых осадках было ошеломляющей новостью для геологов того времени, так как они, подобно упоминавшимся выше авторам, считали, что трещины возникают вследствие тектонических деформаций в уже литифицированных породах. Добавим, что и представления современных геологов, несмотря на очевидные факты, мало изменились. Например, предлагается «рассматривать системы первичных трещин как следствие процессов релаксации, протекавших в пораженных ими объемах среды» [Ponomarev, 2011]. Другим примером могут служить модели, согласно которым первичные трещины в осадочных породах любого возраста имеют неотектоническое происхождение и развиваются в хрупкой среде вдоль оси сжатия (часто вследствие разгрузки и подъема породы на поверхность). Эти модели, предложенные сначала для Северо-Американской платформы [Engelder, 1982], в дальнейшем были распространены и на другие регионы [Hancock, Engelder, 1989; и др.].

Изучая более 30 лет феномен трещиноватости в разных тектонических обстановках и не зная о работах Г.К. Джильберта, мы самостоятельно пришли к выводу о том, что первичная трещиноватость осадочных пород связана с регулярной макроскопической структурой, возникающей в осадках на ранних стадиях их консолидации и диагенеза и представляющей квазирегулярную сеть узких слоев, в которых связность частиц резко понижена. В качестве возможных механизмов образования этой структуры ранее мы предложили рассматривать внутреннюю (т.е. неустранимую с помощью граничных условий) неустойчивость осадков, влекущую за собой локализацию сдвиговых пластических деформаций в узких регулярных слоях [Belousov, Mukhamediev, 1990; Mukhamediev, 1990], а также возникновение макроскопических нарушений, связанных с эволюцией вдоль оси сжатия дефектов упаковки частиц [Mukhamediev, 1990]. В дальнейшем модель претерпела некоторые модификации, но вывод о возникновении макроскопической регулярной структуры в визуально не деформируемых слабосвязанных (loose) осадках при их начальном горизонтальном залегании остался неизменным.

Эта структура наблюдается редко, но, как показывает наш опыт, может проявиться даже при слабом, специально подобранном, внешнем воздействии на молодые, почти рыхлые, осадки, например на влажный песок. Структура не может возникнуть в результате случайной упаковки частиц при седиментации. Действительно, кристаллоподобные кластеры частиц («блоки»), которые образуются при плотной случайной упаковке гранулированной среды, имеют локальный нерегулярный характер [Radin, 2008; Zamponi, 2008]. Для возник- 
новения регулярной структуры, очевидно, требуется некий детерминирующий механизм, обеспечивающий образование дальнего порядка. Интенсивность механизма может быть весьма низкой вследствие слабой связанности системы. Длительность и нестационарность внешнего воздействия еще более снижают требования к интенсивности механизма. По меньшей мере в тектонически спокойной обстановке на роль такого механизма в слое осадков могут претендовать эффекты унаследованности от нижележащих, уже литифицированных, трещиноватых слоев ${ }^{9}$ и/или малые напряжения, обусловленные вращением Земли или нерегулярностью этого вращения. От фактора вращения Земли не следует отказываться a priori, так как в обсуждаемой структуре часто представлены четыре ортогональные к напластованию плоскости, направление двух из которых близко к широтному и долготному, а две другие имеют простирание, примерно диагональное к первым двум.

Нет никаких свидетельств того, что возникающая структура после превращения осадков в горную породу (во всяком случае, неметаморфизованную) исчезает или заменяется какой-либо иной. Она служит своеобразным каркасом для зарождающихся в породе систем естественных трещин, которые обычно классифицируются как первичные. Какие именно из слоев ослабления эволюционируют в наблюдающиеся первичные трещины, зависит как от особенностей протекания процессов консолидации и диагенеза, так и от существующих тектонических условий. Последнее обстоятельство и позволяет, в принципе, ставить вопрос о реконструкции палеонапряжений по направлениям первичных систем трещин. Непроявившиеся слои структуры, тем не менее, в литифицированной породе «замораживаются», задавая латентные направления пониженной прочности. Эти направления могут проявиться как вторичные трещины, возникающие и развивающиеся в хрупкой среде на фоне первичных в результате интенсивного воздействия на литифицированную породу, например при проведении взрывных работ в карьерах [Mukhamediev, Grachev, 2000]. По геометрии вторичных трещин можно реконструировать то напряженное состояние, которое привело к их образованию [Grachev, Mukhamediev, 2010].

Итак, сформировавшаяся горная порода, даже не испытавшая макроскопических деформаций, имеет «изначально заданную» блочную природу, обусловленную наличием систем первичных трещин и/или систем ослабленных поверхностей, опреде-

\footnotetext{
9 Такая точка зрения отражена, например, в работе [Grachev, 2007], в которой причина унаследованности усматривается в воздействии вертикального потока порового флюида в процессе диагенеза.
}

ляющих разрывную анизотропию прочностных свойств. Наличие такой структуры является формой существования литифицированных осадочных пород, их отличительным признаком в любом возрасте, а не результатом процессов деформирования геосреды, ее деградации и разрушения. Для того чтобы опровергнуть наше утверждение, следует решить невыполнимую задачу - предъявить пример конкретного обнажения, где породы лишены описываемых свойств и являют собой на макроуровне изотропный однородный монолит, отвечающий цитированным выше представлениям оппонентов.

\section{6. ОПРОВЕРЖЕНИЕ КРИТИКИ КОНТИНУАЛЬНОЙ МОДЕЛИ}

Критические замечания в адрес континуальной модели, содержащиеся в работах по исследованию геосреды, многочисленны и разнообразны. В настоящем разделе сосредоточимся на критике, вызванной недостаточным знанием современной теории ТДТ и современных подходов к постановкам задач, а также на критике, высказываемой сторонниками концепции так называемой активной среды.

\section{1. О КРИТИКЕ СО СТОРОНЫ ПОСЛЕДОВАТЕЛЕЙ Л.Д. ЛАНДАУ И Е.М. ЛИВШИЦА}

Многие отечественные критики модели сплошной среды ознакомились с этой моделью в лучшем случае по книге [Landau, Lifshitz, 1987], что следует из цитированной в соответствующих работах литературы, и стали отождествлять прочитанное с современным состоянием теории ТДТ. Этому поспособствовали и сами авторы книги, которые в первом же предложении §1 заявили: «Механика твердых тел, рассматриваемых как сплошные среды, составляет содержание теории упругости». Следует сразу отметить, что пособие Л.Д. Ландау и Е.М. Лившица не может претендовать не только на описание модели ТДТ, но даже и на сколько-нибудь широкий охват весьма частного ее случая - упругой модели. Это пособие ограничивается геометрически и физически линейной теорией упругости с нулевыми напряжениями в отсчетном состоянии: «Если тело не деформировано, то внутренние напряжения в нем отсутствуют» [Landau, Lifshitz, 1987, p. 13]. На самом деле, формулы, связывающие приращения напряжений и дисторсии в упругом теле с начальными напряжениями, содержатся уже, по крайней мере, в четвертом издании [Love, 1927], вышедшем на русском языке в 1935 г. Мы не смогли обнаружить ссылок на фундаментальный труд А. Лява в [Landau, Lifshitz, 1987]. 
Таким образом, в обсуждаемой книге рассматривается та модель упругого тела, которую впервые разработал Коши в начале XIX века и которая из-за нулевых напряжений в конфигурации к, вообще говоря, не подходит для описания геосреды, в любой реальной конфигурации существенно находящейся в напряженном состоянии, хотя бы вследствие наличия силы тяжести. Предположение об отсутствии начальных напряжений в первоначальном варианте континуума Коши принято, видимо, из соображений простоты и адекватности при описании относительно небольших стандартных объектов наблюдения. Некорректно предъявлять претензии по поводу отсутствия всеохватности к принципиально новой концепции, вошедшей впоследствии в фундамент физики сплошных сред. К тому же последователи научились в теории ТДТ обходиться без этого частного и непринципиального предположения (см. раздел 3.3). Тем не менее во многих работах геолого-геофизического направления обсуждаемое предположение продолжает ошибочно оцениваться как фундаментальное положение, неразрывно присущее моделям сплошной среды, на основании чего необоснованно критикуется континуальный подход в целом.

Рецензируя первое издание книги Л.Д. Ландау и Е.М. Лившица, В.Л. Гинзбург отмечал «частое отсутствие оговорок об ограниченной применимости приводимых положений и об условиях, когда они не соблюдаются» [Ginzburg, 1946]. К сожалению, это замечание В.Л. Гинзбурга в последующих изданиях книги не было учтено в должной мере. В частности, при изложении теории напряжений не оговариваются предположения, явно или неявно сделанные самим Коши при построении его модели (см. раздел 3.2).

Развивая свою мысль о недостаточном освещении исходных посылок, В.Л. Гинзбург в своей рецензии отмечает, что в книге Л.Д. Ландау и Е.М. Лившица «симметричность тензора напряжений доказывается так, что может создаться впечатление о ее неизбежности». Относительно создаваемого впечатления В.Л. Гинзбург угадал точно и на много лет вперед. И в настоящее время появляются работы, в которых симметричность тензора напряжений объявляется основополагающим физическим принципом. Так, в работе [Vikulin, Ivanchin, 2013], в которой критикуется статья [Garagash, Nikolaevskiy, 2009], а вместе с ней и континуум Коссера в целом, фактически утверждается, что несимметричность тензора напряжений ведет к нарушению закона сохранения момента импульса. В дополнение к этому заявляется, что «вопрос о симметричности тензора напряжений, вообще говоря, является решенным» [Vikulin et al., 2016], но не сообщается, кто автор такого решения, о каком тен- зоре напряжений идет речь и в рамках какого континуума введен этот тензор. С отмеченными утверждениями согласиться нельзя. Действительно, из закона сохранения момента импульса симметричность тензора Коши $\mathbf{T}$ вытекает как следствие при соблюдении тех предположений, которые приняты при построении континуума Коши (см. раздел 3.2). Ослабление некоторых из них (стандартный способ получения в механике Тдт обобщений континуума Коши) может привести к несимметричности тензора силовых напряжений 10 , так как изменяется форма записи закона сохранения момента импульса. Абсолютизация симметричности тензора силовых напряжений неявно диктуется, видимо, убежденностью в том, что помимо континуума Коши другие модельные континуумы создать невозможно.

Подчеркнем, что любые рассуждения о свойствах тензора напряжений беспредметны, если, как это делается в [Vikulin, Ivanchin, 2013; Vikulin et al., 2016] и в других работах, не конкретизируется, какие, собственно, силовые напряжения (Коши, Пиолы, Кирхгофа, Эшелби и т.д.) имеются в виду. Например, тензор напряжений Пиолы $\mathbf{T}_{\kappa}$, введенный соотношением (7), при несовпадении отсчетной и актуальной конфигураций частицы, как было уже отмечено, несимметричен сам по себе, безотносительно к любым физическим законам. Итак, тензор напряжений Коши Т симметричен по определению, а тензор силовых напряжений, не являющийся тензором Коши, может быть несимметричным.

\section{2. О КРИТИКЕ СО СТОРОНЫ ПРИВЕРЖЕНЦЕВ АКТИВНОЙ ГЕОСРЕДЫ}

Иногда в литературе можно встретить рассуждения о «живой Земле» или «активной геосреде». Авторы проводят мысль о невозможности учета подобных свойств в рамках континуального подхода, в том числе и потому, что в этом подходе якобы среда только пассивно реагирует на приложенные граничные усилия. Такая критика не нова. Сравним две цитаты: «Сила у ньютонианцев рассматривалась ... как причина движения ${ }^{11} \ldots$ В этой концепции материя выступает как нечто движимое, пассивное, инертное» и «Деформации геологической среды традиционно рассматривают как результат приложения внешних сил. Поскольку представление об энергетической пассивности среды доминирует ..., оно блокирует изучение сред в состоянии их энер-

\footnotetext{
10 Этот тензор уже нельзя будет назвать тензором Коши.

11 В цитированной фразе содержится типичная ошибка, нередко встречающаяся и в геолого-геофизической литературе: у Ньютона, в отличие от Аристотеля, сила является причиной не движения, а изменения движения.
} 
гетической активности». На первый взгляд приведенные утверждения заимствованы из одного источника. На самом деле цитированные работы разделены более чем 60-летним промежутком. Первая цитата заимствована из статьи [Suvorov, Shteinman, 1950], изложение которой имеет скорее идеологическую, а не научную направленность и в «доказательной» части опирается на непререкаемые авторитеты своего времени (ЦК ВКП(б), Энгельс, Ленин), вторая - из аннотации к работе [Ponomarev, 2011], в которой механика ТДТ представлена как парадигма, препятствующая моделированию дискретных и активных сред.

Не имея возможности из-за ограничений по объему провести развернутое обсуждение роли континуального подхода в изучении активных систем (т.е. систем, частицы которых могут до известной степени самостоятельно преобразовывать запасенную энергию в движение), мы отсылаем читателя за подробностями к обзорным работам (например [Toner et al., 2005; Marchetti et al., 2013; Takatori, Brady, 2016]). Отметим лишь, что континуальная модель используется в физике активных систем отнюдь не только из-за того, что, представляя некоторые системы, состоящие из огромного количества частиц (например, молекул), как дискретные, исследователь сталкивается со значительными трудностями, связанными с эффективностью и точностью вычислений, а также с однозначностью интерпретации результатов. Дело в том, что в рамках модели сплошности весьма удачно описывается кинематика коллективного поведения как живых систем (колоний микроорганизмов in vitro, стай птиц, толп людей и т.д.), так и активных систем других типов, а также явления переноса и неустойчивости. В последние годы появилось понимание и важности напряжений в активных системах [Hawkins, Liverpool, 2014]. Можно привести следующий пример приложения концепции напряжений. Еще в 1952 г. Алан Тьюринг смоделировал процесс морфогенеза клеток (т.е. их организованного пространственного распределения в период роста организма) с использованием континуальных уравнений диффузии так называемых морфогенов [Turing, 1952]. С тех пор, несмотря на продолжающиеся исследования, оставался неясным механизм передачи информации о том, что растущий орган достиг необходимого размера и процесс роста должен быть остановлен. Недавно именно неоднородное поле напряжений, сопровождающее рост органа, было предложено в качестве носителя этой информации [Ambrosi et al., 2015]. Итак, модель сплошной среды самостоятельно или в комбинации с дискретной моделью, вопреки высказываемым мнениям, вполне применима к важным аспектам активных сред.
Возвращаясь к геосреде, заметим, что, несмотря на частые упоминания, понятие ее активности фактически не определено. Нет смысла вводить это понятие, если его основывать, как это делается, например, в [Luk'yanov, 2002], на признаках (наличие внутренней энергии, неоднородностей, памяти, возможность разрушения, фазовых переходов и т.д.), которые традиционно рассматриваются в континуальной теории ТДТ без всякой необходимости обращения к термину «активность». Говоря об активности геосреды и о генезисе этого понятия, некоторые российские авторы [Ponomarev, 2008; Vikulin, 2008] первооткрывателем называют А.В. Пейве, утверждая, что он обнаружил новый механизм движения геосреды. При этом ссылаются на статью [Peive, 1961], где в качестве «главной особенности тектонических движений» выдвигалась следующая: «каждый блок обладает как бы самостоятельной “движущей силой”, заключенной в нем самом».

Мы предлагаем альтернативную трактовку утверждения А.В. Пейве. Оно переоткрывает хорошо забытое старое, а именно концепцию импетуса, развитую в работах Филопона (VI век), ибн Сины (Авиценны, XI век), Буридана (XIV век) и других средневековых мыслителей с целью видоизменения учения Аристотеля. Аристотель предполагал, что насильственное (violent) движение тела (после окончания воздействия на него субъектом, вызвавшим движение) продолжается под влиянием движущей (motive) силы со стороны окружающей среды (воздуха), одновременно оказывающей и сопротивление движению [Aristotle, 1981]. Сторонников теории импетуса, как и сейчас противников модели сплошности, не удовлетворял тезис о действии внешней силы. Под импетусом они понимали некую «силу», которая внутренне присуща движущемуся телу, приобретена им, быть может, от вызвавшего движение субъекта и которая по разным причинам постепенно расходуется в процессе движения. Несмотря на то, что в науке гипотеза импетуса (как и воззрения Аристотеля) впоследствии была вытеснена классической физикой Ньютона, ей посвящено множество исследований по истории физики, а также по основам ее преподавания. Последнее вызвано тем, что очень трудно переучить студентов, которые в подавляющем своем большинстве имеют априорно укоренившиеся наивные представления о силах и движениях, сходные с воззрениями Аристотеля и/или концепцией импетуса.

Обсуждение «активной геосреды» завершим ответом на замечание о том, что в рамках модели сплошности среда является пассивной, так как напряжения и деформации геомассива определяются граничными усилиями. В этом замечании спутаны две разные категории - сама модель и способы по- 
становки задач в рамках этой модели. Действительно, классические постановки задач, требующие выставления а) граничных условий и б) постулирования определяющих соотношений материала, обусловлены особенностями технических приложений, в которых выполнение перечисленных требований обычно не вызывает затруднения. Для геосреды складывается совершенно другая ситуация - постулирование определяющих соотношений и выставление граничных условий являются весьма субъективными процедурами, приводящими, например, к существенно разным результатам реконструкции напряжений для одного и того же региона, если авторы используют классические постановки задач. Зато в случае геосреды по данным натурных индикаторов можно получить информацию, например, об ориентации осей главных напряжения в дискретных точках среды. На основе концепции осей напряжения были развиты эффективные подходы к решению геодинамических проблем, позволяющие радикальным образом избавиться от стандартных атрибутов постановки классических краевых задач. Необходимость в постулировании определяющих соотношений среды отпадает, если по натурным индикаторам построить поле траекторий главных напряжений (ТГН), позволяющее совместно с условиями равновесия сформулировать замкнутую систему дифференциальных уравнений гиперболического типа относительно неизвестных величин главных напряжений [Mukhamediev, 1991]. Использование этой системы дает возможность в геодинамических задачах, не конкретизируя реологию литосферы, восстанавливать неизвестные напряжения на отдельных участках границы (например, в зоне коллизии Африканской и Евразийской плит [Mukhamediev, 2002]). Информация о поле ТГН позволяет отбраковывать недопустимые классы определяющих соотношений геосреды [Mukhamediev, 2015]. Если при заданных ТГН дополнительно постулировать определяющие соотношения, то для статически определимых задач необходимость в выставлении граничных условий отпадает вовсе, задача перестает быть краевой и имеет решение только при совместимости реологии и поля ТГН [Mukhamediev, Galybin, 2004, 2007]. Разработаны алгоритмы, позволяющие при фиксированных определяющих соотношениях разыскивать решения без задания граничных условий непосредственно по дискретным данным об ориентации главных напряжений [Galybin, Mukhamediev, 2004].

Резюмируя круг вопросов, касающихся активной среды, приходим к выводу о том, что в случае геосреды понятие активности не определено и отсутствуют убедительные доводы в пользу его принятия, поэтому умозаключения об активности гео- среды, по меньшей мере, являются дискуссионными. В то же время в рамках континуального подхода разработаны постановки задач, позволяющие находить решения без задания граничных условий, что опровергает мнение о якобы неизбежно пассивном отклике сплошной среды на воздействие внешних усилий. Такие постановки представляют потенциальный интерес для исследования действительно активной среды.

\section{7. НЕКОТОРЫЕ ОГРАНИЧЕНИЯ КОНТИНУАЛЬНОГО подХодА}

Для полноценного использования мощного аппарата механики сплошной среды при описании неоднородных и дискретных материалов необходимо иметь возможность выбрать тот репрезентативный макрообъем, который в дальнейшем принимается за тело-точку эффективной среды. Если такая возможность предоставляется, то континуализацию среды, в том числе и иерархической, можно пытаться провести на основе одной из многочисленных процедур осреднения, предлагавшихся в теории. Следует помнить, что при отсчетном описании в теории ТДТ частицы индивидуализированы. Подразумевается, что лагранжевая частица $\mathbf{x}$ за которой следят в процессе движения, существует и является неделимой, а также имеет в области гладкости отображения (2) одних и тех же соседей, причем в силу непрерывности эти соседи не могут терять контакт с х и меняться местами. При нарушении отмеченных требований строгое и последовательное описание движения среды в рамках модели сплошности сталкивается с принципиальными трудностями.

\section{1. МОДЕЛИ СРЕДЫ С МОБИЛЬНЫМИ ДЕФЕКТАМИ}

В литературе большое внимание уделяется исследованию эволюции микроструктуры геосреды при ее нагружении в связи с прогнозированием характера макроразрушения [Sobolev, Ponomarev, 2003]. В частности, разрабатывается концентрационный критерий, который основан на кинетической теории прочности и в котором используются представления об укрупнении и слиянии дефектов (в случае геосреды - трещин) [Zhurkov et al., 1977] с попытками приложения критерия к прогнозу горных ударов и землетрясений [Zavyalov, 2005; Kuksenko et al., 2010]. Проведенное нами экспериментальное наблюдение перераспределения дефектов по размерам в образцах из прозрачного материала, подверженных постоянной скорости укорочения, показало, что при увеличении общего числа дефектов количество малых дефектов умень- 
шается за счет увеличения их размеров, а количество крупных дефектов растет как за счет возникновения новых дефектов, так и за счет укрупнения малых [Bykov et al., 2014].

Общей особенностью поведения существующих дефектов в отмеченных экспериментах и моделях является то, что они, увеличиваясь в размерах, не меняют своего местоположения в материале - раз оказавшись на границе дефекта, материальный элемент окружающей среды так и останется принадлежащим этой границе. В терминах отображения (2), записанного для исходной среды с дефектами, укрупнение и слияние дефектов означает, что $D\left(t_{1}\right) \subseteq D\left(t_{2}\right)$ при $t_{2}>t_{1}$, где $D(t)-$ многосвязное множество точек $\mathbf{x}$ в теле $B$, в которых функция $\mathbf{r}(\mathbf{x}, t)$ терпит разрыв или потерю гладкости в момент $t$. Переход к эффективной сплошной среде производится стандартным способом, в результате чего репрезентативный макрообъем $\mathbf{x}^{\text {eff }}$ включает в себя множество дефектов. В рамках так называемой континуальной теории разрушения [Kondaurov et al., 1987] факт наличия распределенных изменяющихся дефектов в макрообъеме хеff учитывается феноменологически на основе введения в модель эффективной среды скалярного или тензорного параметра, который интерпретируется как мера поврежденности. В энергетическом варианте теории [Kondaurov, 1986] можно определить момент наступления внутренней неустойчивости, но, как и в других вариантах теории, не удается описать эффект локализации поврежденности. Это условие приходится постулировать (например, задавая критические значения меры поврежденности или ее изменения), подобно тому, как в теории прочности и в концентрационном критерии постулируются, соответственно, критические напряжения и критические концентрации трещин (отношение среднего расстояния между ними к их среднему размеру).

Возможность применения континуального подхода к вышеописанному процессу эволюции системы дефектов кроется в том, что в течение всего процесса деформирования макрообъем $\mathbf{x}^{\text {eff }}$ содержит неизменное множество материальных частиц $\mathbf{x}$ исходной среды. Необходимые условия, предъявляемые к $\mathbf{x}^{e f f}$ как к лагранжевой частице, могут быть выполнены. Ситуация кардинально изменится, если дефекту предоставлена возможность двигаться как целому относительно частиц $\mathbf{x}$ исходной среды. Такие дефекты мы называем мобильными. В процессе движения частицы $\mathbf{x}$ исходной среды, окружающие мобильный дефект, с течением времени полностью заменяются другими частицами. Примером мобильного дефекта, движение которого в геосреде не сопровождается массообменом, является сейсмический разрыв, развивающийся как череда динамических подвижек-пульсаций по относительно узким зонам (self-healing slip pulses): каждая такая подвижка инициирует подвижку в смежной зоне, а сама через короткое время останавливается («залечивается») [Heaton, 1990].

Трудности с использованием континуального подхода возникают, если мобильные дефекты имеют объем, и поэтому их движение необходимо сопровождается массообменом. В качестве примера рассмотрим дефекты упаковки частиц (поры) в слабосвязанных гранулированных осадках. При нагружении осадков поры начинают взаимодействовать друг с другом. В частности, две поры, расположенные вдоль оси максимального сжатия, подвержены силам взаимного отталкивания (в смысле Дж. Эшелби [Eshelby, 1970]), а при их ориентации вдоль минимального сжатия поры испытывают силы взаимного притяжения [Mukhamediev, 1990]. Если сцепление между частицами преодолено, то начинается движение, при котором пространство поры замещается частицами, а сама пора перемещается в новое положение, приобретая в свое окружение новые частицы. Схожий механизм миграции пор зачастую является основным при высоких градиентах температуры и напряжений (например, в работающих тепловыделяющих элементах ядерных реакторов [Gill, 2009]), но для гранулированной среды в нормальных условиях такие перескоки дефектов упаковки, которые диктуются стремлением системы понизить свою полную потенциальную энергию, классифицируются как редкие события [Richard et al., 2005]. Тем не менее именно они определяют так называемую медленную динамику гранулированной среды, в частности особенности процесса перехода от рыхлой к плотной случайной упаковке.

Можно ли такой процесс миграции мобильных дефектов смоделировать, оставаясь в рамках отсчетного описания и концепции единой (а не, скажем, двухфазной) сплошной среды? На этот вопрос приходится дать отрицательный ответ. Действительно, какой бы репрезентативный макрообъем $\mathbf{x}^{e f f}$ в эффективной среде ни выбрать, он, в обеспечение миграции пор, рано или поздно потеряет или приобретет материальные частицы исходной среды. Невозможность сохранения массы лагранжевой частицей $\mathbf{x}^{e f f}$ эффективной среды ведет к нарушению других законов сохранения и вызывает необходимость для описания движения мобильных дефектов в сплошной матрице использовать дискретный подход. Этот подход, который в чем-то аналогичен подходам в атомистической модели, описанной в разделе 4.1, был применен к среде со случайным распределением пор в пространстве [Mukhamediev, Ul'kin, 2011, 2014]. В результате расчетов выявлено, что поры в результате их мигра- 
ции образуют относительно регулярную структуру квазипараллельных линейных образований, вытянутых вдоль оси сжатия. Такие образования мы ассоциируем с наблюдающимися в осадочных породах системами слоев повышенной пористости [Du Bernard et al., 2002].

\section{2. МОДЕЛИ СРЕДЫ С ИЕРАРХИЧЕСКИМ ДВИЖЕНИЕМ НА ЛЮБОМ УРОВНЕ}

Рассмотрим модель иерархического тела $B$, в котором любая его часть $\left(B^{*}, B^{*} \subset B\right)$ является неоднородной, а именно содержит ослабленные поверхности раздела, сдвиги или отрывы по которым вызывают деформацию материала. Проще говоря, от наличия неоднородностей этого типа невозможно избавиться ни на каком масштабе, в том числе и в процессе мысленного уменьшения вплоть до 0 размера материальных частиц 12 . В частных случаях при определенных условиях самоподобия введенная иерархическая структура может быть фрактальной или мультифрактальной. При движении рассматриваемого тела любое его подтело, занимавшее при $t=0$ односвязную область в пространстве, в некоторый момент может вследствие разрывных деформаций потерять связность или, как минимум, непрерывность отображения $\mathbf{x} \rightarrow \mathbf{r}$. Отсюда гладкое отображение (2) для $0<t<\infty$ невозможно задать на любом масштабе материала, что означает невозможность введения понятия лагранжевой точки $\mathbf{x}$ в в том числе и в смысле репрезентативного макрообъема $\mathbf{x}^{e f f}$ эффективной сплошной среды. Мы не можем ни на каком масштабе назначить некий материальный объем элементарным, приписав ему некоторое конечное число степеней свободы и конечное число характеристик, которые отражали бы его напряженно-деформированное состояние, так как таких чисел и таких характеристик у него бесконечно много. Концепция отсчетного описания в континуальном подходе теряет силу, а вместе с ней пропадает и возможность записывать балансовые соотношения для материального объема. Представляются нереальными попытки вводить силовые напряжения как тензоры второго ранга.

В геомеханике условие самоподобия структуры, накладывающее ограничения на законы иерархичности, дает возможность продвинуться в теоретическом моделировании некоторых свойств среды [Rodionov et al., 1989], не затрагивая отмеченных сложностей с отсчетным описанием. Если самоподобие выполняется на любом масштабе, то приходим к модели фрактальной (точнее, монофрактальной) среды, которая может быть представлена

\footnotetext{
12 Возражения о пределе делимости, налагаемом существованием атомов и молекул, несостоятельны, т.к. речь идет не о реальном строении среды, а о ее модели.
}

как непрерывный ряд континуумов, определенных характерным размером осреднения $l$. Эффективные характеристики континуумов зависят от $l$ степенным образом с единым показателем степени [Dyskin, 2004]. В последние годы сделаны попытки континуализации фрактальной среды на основе использования математического аппарата дробного дифференцирования и интегрирования [ $L i$, Ostoja-Starzewski, 2011; Drapaca, Sivaloganathan, 2012; Tarasov, 2015] ${ }^{13}$. На наш взгляд, пока в результатах этих попыток формально-математического смысла больше, нежели физического. То же самое можно сказать и о попытках введения силового тензора напряжений в рамках модели фрактальной среды. Достаточно отметить, что размерность «напряжений» оказывается дробной, при этом среди авторов, вводящих «напряжения», существуют принципиальные разногласия (ср., например, [Carpinteri et al., 2007] и [Bažant, Yavari, 2007]).

\section{8. ГЕНЕЗИС ТИПИЧНЫХ ОШИБОК И НЕИСПОЛЬЗОВАННЫЕ ВОЗМОЖНОСТИ КОНТИНУАЛЬНОГО ПОДХОДА ПРИ МОДЕЛИРОВАНИИ ГЕОСРЕДЫ}

\section{1. О ТЕРМИНАХ И ПОНЯТИЯХ, ЗАИМСТВОВАННЫХ ГЕОЛОГИЕЙ ИЗ ФИЗИКИ}

Геология является исторической наукой и по способу познания окружающего мира существенно отличается от физики во многих отношениях. Это приводит к несоответствию понятийных основ и терминологии в этих науках. Для решения рассматриваемой нами задачи построения модели движения геосреды непосредственно геологических понятий и терминов недостаточно, поэтому геология заимствует их из физики. При этом даже возникает дискуссия на тему «какие категории ближе геологии?» [Vikulin, 2013]. Среди кандидатов на заимствование обсуждаются и/или предлагаются нелинейность, фрактальность, энергонасыщенность. Этот список можно расширить, включив в него уже прочно вошедшие в геологию термины, такие, как сила, напряжение, устойчивость и другие.

Нам представляется, что сама постановка вопроса в отмеченной дискуссии не совсем корректна, а призывы к заимствованию могут привести к непрогнозируемым последствиям. Термин обычно заимствуется геологией подобно тому, как в естественном языке заимствуется иностранное слово, т.е. без того исторического и смыслового окружения, которое термин имел в исходном языке (в

\footnotetext{
13 С основами этого аппарата можно ознакомиться, например, по книге [Samko et al., 1987].
} 
нашем случае - в физике). Как следствие, термин либо переосмысливается средствами геологии с вытекающим отсюда искажением своей понятийной основы, либо теряет свое точное определение, либо, наконец, не в достаточной мере понимается. Так, термин нелинейность означает понятие более высокого смыслового уровня, чем противопоставляемое понятие линейности. Тем не менее, как показывает наша практика, геологи, охотно рассуждающие о нелинейности, часто не могут сформулировать, что такое линейность, принимают линейную задачу за нелинейную, если результатом решения этой задачи являются нелинейные зависимости интересующих их величин, и, наоборот, нелинейную задачу - за линейную, если эти зависимости линейны, путают линейность с устойчивостью и т.д.

В качестве примера, плохо определенного и поэтому потенциально вредоносного, рассмотрим понятие «энергонасыщенность», активно внедряемое в научный обиход, в частности, в работах [Ponomarev, 2008, 2011]. Во-первых, это понятие вводится В.С. Пономаревым для удивительного класса сред, в которых упругая энергия и линейна по деформациям $\varepsilon$, так как в цитированных работах принято, что $u\left(\varepsilon_{1}+\varepsilon_{2}\right)=u\left(\varepsilon_{1}\right)+u\left(\varepsilon_{2}\right)$. Такая линейность может иметь место, если напряжения в среде не зависят от деформаций, но тогда об упругости говорить не приходится. При этом невозможно выполнить требование линейности напряжений по деформациям, также выдвигаемое В.С. Пономаревым. Во-вторых, неясно, о какой энергии в текстах B.C. Пономарева вообще идет речь: об упругой энергии системы или о плотности этой энергии? Если справедливо второе предположение, то как рассчитывается эта плотность: на единицу массы или на единицу объема (и в какой конфигурации)? А быть может, имеется в виду полная потенциальная энергия упругой системы в поле силы тяжести?

Это не праздные вопросы. Путаница здесь может привести к принципиальным ошибкам. Рассмотрим следующую задачу. Пусть даны два одинаковых сосуда, в каждый из которых наливается одинаковая масса жидкости одинаковой плотности $\rho_{\kappa}$. Разница в том, что в сосуде 1 жидкость несжимаема, а в сосуде 2 - упруго сжимаема, т.е. имеет конечное значение модуля объемного сжатия $K$. Спрашивается: в каком из сосудов под воздействием силы тяжести потенциальная энергия покоящейся жидкости будет больше? Рассуждения в духе концепции «энергонасыщенности» в качестве правильного ответа указывают на сосуд 2 - ведь жидкость в этом сосуде, помимо гравитационной энергии, обладает («насыщена») и энергией упругого сжатия. Основываясь на таких представлениях, об упругой сжимаемости стали говорить как о «ранее неучтенном источнике энергии», который поставляет «избыточную энергию упругих деформаций» в гидростатически сжатых слоях земной коры и который самостоятельно влияет на устойчивость или неустойчивость этих слоев (см., например, [Rebetsky, 2013, 2014]). На самом деле правильный ответ в задаче иной: в сосуде 2 потенциальная энергия жидкости меньше, чем в сосуде 1, так как, вследствие уменьшения высоты столба по сравнению с несжимаемой жидкостью, сжимаемая жидкость теряет гравитационную энергию в большей степени, чем накапливает упругую. Это утверждение справедливо и для твердых веществ любой реологии, находящихся в состоянии гидростатического сжатия. Величины $K$ в слоях, сами по себе, на устойчивость или неустойчивость не влияют. Единственным источником неустойчивости в системе слоев однородных упругосжимаемых (вязких или невязких) жидкостей является инверсия плотности на границе слоев в актуальном состоянии. Если, к тому же, вещество внутри слоев стратифицировано, то возникает дополнительный источник неустойчивости, связанный с критическим значением вертикального градиента плотности [Mukhamediev et al., 2016].

\section{2. ГЕНЕЗИС ВОСПРОИЗВОДСТВА ДОНЬЮТОНОВСКИХ ВОЗЗРЕНИЙ В ГЕОЛОГИИ}

Изменение первоначального смысла происходит и для терминов, употребляемых в геологии уже длительное время. Пожалуй, наиболее значительный урон геологии и геофизике наносит искажение смысла терминов, связанных с понятиями силы и напряжения ${ }^{14}$. Геосреда являет исследователю свои свойства посредством демонстрации собственной структуры и кинематики движения. Тем не менее очень часто результаты наблюдения геолог докладывает в терминах теории сил и напряжений, а не в терминах наблюдаемых и/или измеряемых перемещений и деформаций. Например, наблюдаемое направление перемещения блока геосреды интерпретируется в терминах направления силы, действующей на него, а о наблюдаемом удлинении или укорочении сообщается, соответственно, как о растяжении или сжатии ${ }^{15}$. Последнее можно делать, например, для идеально-диссипативной среды, рассмотренной в разделе 3.3, но не в случае, когда вли-

\footnotetext{
14 Термины «сила» и «напряжение» заимствованы из механики многими областями знания, но обычно изменение их смысла оговаривается. Так, в медицине под стрессом понимают специфическую реакцию организма на воздействие неблагоприятных факторов. В геологии же неявно полагается, что первоначальный смысл этих терминов не изменен.

15 Другие примеры некорректного употребления динамических терминов взамен кинематических можно найти в [Marrett, Peacock, 1999; Mukhamediev, 2014].
} 
яние начальных напряжений существенно. Неправомерность отождествления кинематических и динамических величин опровергается не только теоретическими аргументами, но и геофизическими примерами. Так, деформации разгрузки, которые возникли после главного толчка в эпицентральной зоне Рачинского землетрясения 1991 г. на юге Большого Кавказа, вызвали удлинение вдоль действующего регионального напряжения сжатия [Belousov et al., 1993].

Если бы дело касалось просто терминологической путаницы, то сложившееся положение можно было выправить, последовав рецепту Конфуция, данному им в 13-й главе книги Лунь Юй: «Необходимо исправить имена» [Confucius, 2006]. К сожалению, этого недостаточно. Сила и напряжение являются понятиями высокой степени абстрактности, их можно измерять лишь косвенно. Тем не менее соблазн связать силы и напряжения непосредственно с наблюдениями возникает перманентно. Видимо, такие попытки будут предприниматься вновь и вновь в самых неожиданных приложениях. Часто геолог действительно полагает, что о направлении силы или о знаке напряжения он может судить по наблюдаемому перемещению или рассчитанной деформации, исходя из соображений «здравого смысла» либо имея набор некоторых априорных правил.

По нашему мнению, возникшая ситуация корнями уходит в некоторые специфические особенности геологии как науки. Так, геолог привык работать с одной системой отсчета, которая задается жестким телом, покоящимся на дневной поверхности в локальной точке наблюдения, а также с выделенной системой координат, в которой ось аппликат вертикальна и относительно которой формулируются некоторые геологические законы (например, литологические) и во многом строится терминология (например, классификация разломов). При анализе нелокальных данных геолог все равно ищет некую систему отсчета, относительно которой следует определять скорости, и пытается придать ей смысл абсолютной системы, поэтому геолог далек от проблем физики, для которой выбор конкретной системы координат вообще не имеет теоретического значения, а система отсчета не является той построенной раз и навсегда сценой, на которой только и разворачиваются события. В классической физике при формулировке законов выдвигается условие инвариантности этих законов относительно замены координат и, как минимум, независимости их от выбора инерциальной системы отсчета. При этом преобразования, связанные с изменениями координат и системы отсчета, используются не только для проверки корректности законов, но и для получения этих законов на основе симметрий с использованием принципа наименьшего действия. Именно так, на основе симметрий физического пространства и времени, можно получить законы сохранения импульса, момента импульса и энергии, используя знаменитую теорему Эмми Нетер [Noether, 1918]. Симметрии однородного изотропного вещества позволили на основе теоремы Нетер сформулировать законы сохранения импульса и момента импульса для материального пространства геосреды, дуального физическому [Mukhamediev, 1990].

Наличие законов сохранения есть главная черта современной классической физики, отличающая ее от доньютоновского естествознания. В рамках воззрений Аристотеля подобных законов получить не удается [Disy, Garner, 1999]. В геологии же, ориентированной на незыблемость систем отсчета и координат, отношение к законам сохранения, по меньшей мере, равнодушное - ведь открытие этих законов связано с драмой идей совсем в другой науке, а само их существование или отсутствие никак не отражается на способе геологического познания мира. Вследствие этого геолог, который не приемлет инородных для него законов, при интерпретации кинематики в терминах динамических величин полагается не на физику, а на геометрию. В отношении сил и движений это означает, что геолог совмещает наблюдаемое направление движения с искомым направлением силы. А совпадение этих направлений и есть одно из важнейших положений Аристотеля, выдвинутых им при исследовании насильственного движения [Aristotle, 1981].

При таком образе действий геолог вправе рассчитывать на получение корректного результата в случае, когда блок совершает установившееся движение в вязко сопротивляющейся среде. В этом асимптотическом случае направление вынуждающей (в терминологии геолога - активной) силы $\mathbf{f}$ будет совпадать с направлением перемещения $\delta \mathbf{u}$ не только у Аристотеля, но и у Ньютона. Если на движение блока накладывается единственная голономная связь (например, описывающая поверхность блока 2 , по которой исходный блок 1 совершает установившееся контактное скольжение с вязким трением - асейсмическое криповое движение), то сказанное выше о совпадении направлений остается справедливым, только под силой $\mathbf{f}$ следует понимать ее проекцию $\mathbf{f}_{\tau}$ на поверхность связи, а под перемещением $\delta \mathbf{u}-$ относительное перемещение блоков $[\delta \mathbf{u}]^{16}$.

Однако, если условия движения и природа сил

16 Постулат Аристотеля о совпадении по направлению векторов $\mathbf{f}_{\tau}$ и $[\delta \mathbf{u}]$ в геолого-геофизической литературе стали почему-то приписывать авторам работ [Wallace, 1951; Bott, 1959; Gushchenko, 1973]. 
сопротивления иные 17 , то трогательная картина единения Аристотеля и Ньютона рассыпается. Пусть, например, взаимное движение блоков 1 и 2 происходит сейсмическим образом. Для Аристотеля такая смена типа движения не повлияет на его утверждение о сонаправленности векторов $\mathbf{f}_{\tau}$ и

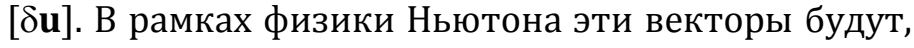
вообще говоря, разнонаправленны. Для определения угла между их направлениями следует решать уравнения движения с учетом сил инерции и сопротивления при заданных начальных условиях.

\section{3. ИГНОРИРОВАНИЕ ФИЗИКИ В МЕТОДАХ РЕКОНСТРУКЦИИ НАПРЯЖЕНИЙ ПО КИНЕМАТИЧЕСКИМ ДАННЫМ}

Рассмотрим важную как для фундаментального знания, так и для приложений проблему определения поля напряжений геосреды по разрывным необратимым сдвигам, фиксируемым по механизмам очагов землетрясений, по ориентации борозд скольжения и по другим кинематическим данным. Макроскопическое поле скорости деформации в эффективной среде $\dot{\mathbf{E}}\left(\mathbf{r}^{e f f}, \mathrm{t}\right) \quad\left(\mathbf{r}^{e f f}=\mathbf{r}\left(\mathbf{x}^{\text {eff }}, t\right)\right)$ можно рассчитать, используя для каждого пространственного макрообъема reff формулы (6), (13). При этом, естественно, для каждого $i$-того сдвига помимо непосредственно наблюдаемого его направления необходимо знать величину $[\delta u]_{(i)} d s_{(i)}$, где первый сомножитель - это амплитуда подвижки, а второй - площадь, по которой она произошла. Для определения величин $[\delta u]_{(i)} d s_{(i)}$ по сейсмологическим данным имеет смысл использовать эмпирические зависимости связи сомножителей с магнитудой землетрясений (например, из [Wells, Coppersmith, 1994] или более новые). Со скейлинговыми соотношениями для разломов, основанными на геологических данных, можно ознакомиться по обзору [Torabi, Berg, 2011]. Если, не домысливая дополнительных сущностей, ограничиться только наблюдаемыми величинами, то при континуализации геосреды, деформируемой необратимыми разрывными сдвигами, форсируемым образом приходим к модели идеально-диссипативной среды ${ }^{18}$, рассмотренной в разделе 3.3 .

Задача сводится к определению в этой среде макроскопического поля тензора напряжений Ко-

\footnotetext{
17 Предположение об установившемся движении с вязким трением опровергается наблюдаемым наличием борозд скольжения и других микроповреждений на поверхностях взаимодействующих блоков.

18 Предположение о мгновенно затухающей памяти приходится принимать, т.к. обычно используются данные о сдвигах за единственный интервал времени $\Delta t$. Модель диссипативной среды можно обобщить на учет памяти, если есть возможность использования данных по ряду последовательных интервалов $\Delta t_{1}, \Delta t_{2}, \ldots$
}

ши $\mathbf{T}\left(\mathbf{r}^{e f f}, t\right)$ по известному полю $\dot{\mathbf{E}}\left(\mathbf{r}^{e f f}, t\right)$. При решении задачи неизбежно возникает дилемма:

1) использовать законы сохранения или

2) выдвинуть какие-либо априорные предположения об определяющих соотношениях геосреды.

Сформулированные подходы являются взаимоисключающими. В отличие от того, как это делается при классических постановках модельных краевых задач механики ТДТ, где оба поля - T и $\dot{\mathbf{E}}-$ являются искомыми, в случае заданного поля $\dot{\mathbf{E}}$ эти подходы уже нельзя использовать совместно. Первый подход опирается на учет объективных законов природы. Второй является субъективным, так как в отличие от экспериментальной механики ТДТ, где определяющие соотношения выявляются на основе многочисленных испытаний образцов материала, в случае геосреды эти соотношения задаются умозрительно, о чем уже упоминалось в разделе 6.2.

Сложилось так, что представители геологогеофизического сообщества уже в течение нескольких десятилетий предпочитают именно второй подход, даже не рассматривая возможности первого. Ими выдвинуты разного рода весьма специфические «определяющие соотношения», которые сводятся к связи искомого тензора Т с наблюдаемыми в макрообъеме микродисторсиями $d \mathbf{s}_{(i)} \otimes[\delta \mathbf{u}]_{(i)} / d V, i=1, \ldots, N$. Предложены разные модификации, отличающиеся друг от друга выбором постулируемой зависимости Т от направлений подвижек. Например, часто предполагается совпадение на площадках разрыва $d \mathbf{s}_{(i)}=\mathbf{n}_{(i)} d s_{(i)}$ направлений вектора относительной микроскопической подвижки $[\delta \mathbf{u}]_{(i)}$ и макроскопического вектора силы $\mathbf{f}_{\tau(i)}^{\text {macr }}=d \mathbf{s}_{(i)} \cdot \mathbf{T} \cdot\left(\mathbf{I}-\mathbf{n}_{(i)} \otimes \mathbf{n}_{(i)}\right)$. Если бы к сравнению привлекался реально действующий на площадке $d \mathbf{s}_{(i)}$ микроскопический вектор силы $\mathbf{f}_{\tau(i)}$, то мы имели бы дело с постулатом Аристотеля. Замена $\mathbf{f}_{\tau(i)}$ на $\mathbf{f}_{\tau(i)}^{\text {macr }}$ усугубляет некорректность выдвинутого предположения. Рассогласование направлений $[\delta \mathbf{u}]_{(i)}$ и $\mathbf{f}_{\tau(i)}^{\text {macr }}$ будет возникать не только по причинам, рассмотренным в конце раздела 8.2, но и в самом благоприятном случае нестесненной квазистатической подвижки по единственной площадке разрыва. Угол между $[\delta \mathbf{u}]_{(i)}$ и $\mathbf{f}_{\tau(i)}^{\operatorname{macr}}$ в упругой среде определяется формой площадки [Kostrov, Das, 1984].

Важно отметить два обстоятельства, связанные с модификациями, которые существуют в рамках второго подхода. Во-первых, автор каждой модификации, выдвинув свое личное «определяющее соотношение», считает его универсальным и применяет к массиву геосреды любой природы, не обращая внимания, в частности, на тип и структуру слагающих массив пород. Такой образ действий резко контрастирует с тем, что принято в механи- 
ке, где каждый материал характеризуется своими собственными определяющими соотношениями, отличающими его от других материалов. Вовторых, с точки зрения физики все различия в модификациях непринципиальны. Какие бы замысловатые ни выдвигались априорные постулаты, связывающие в $\mathbf{r}$ eff искомый тензор $\mathbf{T}$ с наблюдаемой кинематикой, их наличие не оставляет места для выполнения законов сохранения в эффективной среде, и, следовательно, во втором из подходов физика игнорируется ${ }^{19}$. Невыполнение закона сохранения импульса, который сводится к уравнению равновесия (см. первое соотношение (10)) и который является необходимым условием существования тензора Коши $\mathbf{T}$, означает, что в рамках второго подхода реконструируются величины, которые заведомо к напряжениям никакого отношения не имеют ${ }^{20}$. Вследствие того, что условие $\mathbf{T}=\mathbf{T}^{T}$ в первом соотношении (11) имеет место только при выполнении закона сохранения импульса, ясно, что априорно принимаемое в рамках второго подхода предположение о симметричности тензора Т ни на чем не основано.

Впервые на невыполнение условий равновесия в обсуждаемом подходе было указано в работе [Mukhamediev, 1993], нарушение аксиом теории определяющих соотношений было проанализировано в [Mukhamediev, Brady, 2002], а исследование нарушения закона сохранения импульса - в [Mukhamediev, 2014]. К сожалению, именно этот подход стал сейчас доминирующим. Вряд ли на Земле остался регион, для которого, при наличии хотя бы минимального набора необходимых данных, не предпринимались попытки рассчитать «напряжения» и «палеонапряжения» на его основе. Этот подход нашел широкое отражение в изданиях геологогеофизической направленности.

\section{4. ОБ УЧЕТЕ ЗАКОНОВ СОХРАНЕНИЯ В МЕТОДАХ РЕКОНСТРУКЦИИ НАПРЯЖЕНИЙ ПО КИНЕМАТИЧЕСКИМ ДАННЫМ}

Вернемся к сформулированной в разделе 8.3 дилемме относительно реконструкции поля $\mathbf{T}\left(\mathbf{r}^{e f f}, t\right)$ по известному полю $\dot{\mathbf{E}}\left(\mathbf{r}^{e f f}, t\right)$. Возникают вопросы. Почему сторонники второго подхода не постулируют связь макроскопической силовой характери-

\footnotetext{
19 Некоторые дополнительные подробности и разъяснения по этому поводу приведены в разделе 8.4.

20 На основе этого подхода в макрообъеме определяются четыре скалярные величины, которые авторы интерпретируют как элементы редуцированного тензора напряжений $\mathbf{T}_{R}$. В peдуцированном тензоре $\mathbf{T}_{R}$ не определен первый инвариант $I_{1}$ и произвольно нормирован второй инвариант $I_{2}$, так что каждый тензор $\mathbf{T}_{R}$ характеризуется четырьмя независимыми скалярными параметрами, а не шестью, как исходный нередуцированный тензор Коши т.
}

стики $\mathbf{T}$ с макроскопической же кинематической характеристикой $\dot{\mathbf{E}}$ ? Ведь именно $\mathbf{T}$ и $\dot{\mathbf{E}}$ являются термодинамически сопряженными величинами, так как их свертка, T: $\dot{\mathbf{E}}$, определяет локальную скорость совершения внутренней работы. Почему, нарушая аксиому макроскопической определимости в теории определяющих соотношений и требования термодинамики, авторы постулируют зависимость Т от направлений подвижек, т.е. от микроскопических, с точки зрения эффективной среды, характеристик, к тому же не имеющих никакого термодинамического смысла?

Прежде чем ответить на этот вопрос, проанализируем, что произойдет, если мы постулируем какую-либо (корректную с точки зрения теории определяющих соотношений) взаимосвязь тензоров $\mathbf{T}$ и $\dot{\mathbf{E}}$ ? Пусть, например, мы предполагаем выполнение определяющего соотношения (14) и, естественно, требуем, чтобы в деформируемой среде выполнялись законы сохранения. Тогда, подставляя (14) в первое из соотношений (10) и учитывая, что в несжимаемой среде $\rho=$ const, обнаруживаем, что на тензорную функцию $\dot{\mathbf{E}}\left(\mathbf{r}^{e f f}, t\right)$ накладывается ограничение $\nabla \times(\nabla F \cdot \dot{\mathbf{E}}+F \nabla \cdot \dot{\mathbf{E}})=0$ [Mukhamediev, 2015]. Выбрав другое определяющее соотношение, получим другое ограничение. Но поле $\dot{\mathbf{E}}\left(\mathbf{r}^{e f f}, t\right)$ предъявляется природой, которая от наблюдателя не зависит. Это поле абсолютно произвольно, на него нельзя накладывать никаких априорных ограничений. Вскрытое противоречие ясно указывает на неправомерность действий, которые направлены на субъективное приписывание геосреде каких-либо связей между $\mathbf{T}$ и $\mathbf{E}$ и которые, тем самым, нарушают законы сохранения.

Теперь можно ответить на вопросы, поставленные в начале раздела. Если бы авторы постулировали взаимосвязь между $\mathbf{T}$ и $\dot{\mathbf{E}}$, то факт нарушения условий равновесия вскрывался бы легко и обсуждаемый второй подход к решению сформулированной дилеммы просуществовал бы недолго. Постулирование же связи тензора напряжений с направлениями подвижек на площадках сдвига, ничего не меняя по существу, в значительной мере маскирует неправомерность субъективных процедур. Внимание неискушенного пользователя или читателя отвлекается от принципиальных проблем и перенаправляется на вопрос, связанный с зави-

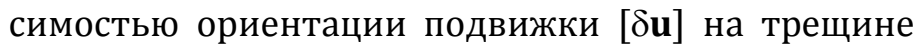
или разломе от действующего напряжения. При этом авторы методологических работ представляют дело так, как будто этот вопрос является наиважнейшим в процедуре реконструкции $\mathbf{T}$ и как будто у исследователя есть достаточно произвола, чтобы закрыть этот вопрос с помощью разного рода предположений, например типа постулата 
Аристотеля. Не случайно в этих работах не приводится корректное определение искомого объекта - тензора Т. У каждого из авторов какой-либо модификации этот «тензор» свой, он зависит от выдвинутых предположений. Эти тензоры объединяет лишь одно общее свойство - они не имеют отношения к тензору T, вводимому в континууме Коши.

Первый из двух подходов в дилемме опирается на учет законов сохранения, роль которых в данном случае выполняют первые соотношения в (10) и (11). Рассмотрим возможный алгоритм реализации подхода для идеально-диссипативной геосреды. Так как и в классе вязких сред, задаваемых определяющим соотношением (14), и в классе пластических сред (15) главные оси искомого тензора напряжений Т совпадают (и одинаково пронумерованы по признаку возрастания главных значений) с осями тензора $\dot{\mathbf{E}}$, по известному полю $\dot{\mathbf{E}}\left(\mathbf{r}^{e f f}, t\right)$ в исследуемой области получаем поле траекторий главных напряжений (ТГН). Приходим к поставленной в [Mukhamediev, 1991] задаче: на основе интегрирования уравнений равновесия определить поле напряжений по полю ТГН при корректно поставленных граничных условиях в напряжениях без постулирования определяющих соотношений. В результате решения этой задачи в исследуемой области реконструируется поле $\mathbf{T}\left(\mathbf{r}^{e f f}, t\right)$. Этот результат отличается от результата, получаемого в рамках второго подхода. Вопервых, определяется поле полного тензора напряжений, т.е. шесть скалярных полей, а не четыре. Во-вторых, определяемое поле $\mathbf{T}\left(\mathbf{r}^{e f f}, t\right)$ равновесно, что позволяет интерпретировать его как приближение к реально существующему в геосреде полю напряжений.

На текущем этапе использованы лишь данные о распределении ориентации главных осей в заданном кинематическом поле $\dot{\mathbf{E}}\left(\mathbf{r}^{e f f}, t\right)$. Не использованные пока данные о распределении величин скоростей деформации позволяют в рамках заранее фиксированного класса реологических моделей геосреды конкретизировать реальные определяющие соотношения. Например, алгоритмы определения изотропного пластического потенциала $\psi\left(I_{2}\left(\mathbf{T}^{d e v}\right), I_{3}\left(\mathbf{T}^{d e v}\right)\right)$ в классе пластических моделей приведены в работах [Mukhamediev, 1993; Mukhamediev, Brady, 2002]. Получаемый пластический потенциал можно интерпретировать как сейсмотектонический потенциал исследуемого региона. Таким образом, в рамках первого подхода появляется возможность не только корректно учесть законы сохранения, но и получить полную информацию о поле напряжений и приближенно оценить реологические свойства геосреды.

\section{9. ЗАКЛЮЧЕНИЕ}

Настоящая работа посвящена обсуждению строения геосреды, т.е. доступных для изучения литифицированных горных пород, и способов описания движения этой среды. Отмечается, что в рамках затронутого круга вопросов существуют два мнения: 1) изначально однородная сплошная геосреда приобретает свойство блочности в процессе ее деформирования (а также деструкции, деградации, разрушения) и 2) геосреда является блочной (а нередко, помимо этого, иерархической, активной, энергонасыщенной), и поэтому модель сплошности недопустима при описании ее деформирования. Сторонники первой точки зрения активно применяют континуальную модель твердого деформируемого тела в расчетах напряженно-деформированного состояния. Модель используется либо стандартная, либо тем или иным способом модифицированная, но входные параметры модели принципиально не содержат сведения о дискретности. Авторы, придерживающиеся второго мнения, напротив, модель ТДТ отвергают, явно или неявно полагая, что блочная структура геосреды, выявляемая геологическими методами, прямым и однозначным образом влияет на все ее иные механические свойства и, в первую очередь, на характер ее движения.

Мы выражаем определенное несогласие с обеими отмеченными точками зрения. Первая из них опирается на представление о том, что изначально в геосреде отсутствует какая-либо структура, а процессом приобретения дискретной структуры геосреда обязана тому, что впоследствии вовлекается в некие деформационные процессы. Наши возражения опираются на длительный опыт полевых работ, в течение которых мы никогда не наблюдали бесструктурных горных пород, а, напротив, обнаруживали наличие регулярной блочной структуры даже в еще очень молодых недеформированных осадках. Мы приходим к выводу, что наличие квазирегулярной дискретной структуры является не приобретенным свойством, а формой существования геосреды и причиной анизотропии ее прочностных характеристик. При последующих деформациях геосреды те или иные элементы врожденной структуры могут выявиться четче, сама структура может обогатиться за счет вторичных эффектов.

В дискуссии со сторонниками второй из отмеченных выше точек зрения мы исходим из того, что модель движения геосреды не обязана точно копировать все подробности ее геологического строения, тем более, что даже экспериментально определяемые особенности деформации геосреды не отражают ее структуру однозначным и прямым образом. В частности, изменчивые во времени бло- 
ки, выделяемые по данным GPS-измерений в поле современных скоростей, не копируют консервативную структуру геологических блоков, но в то же время на смежном масштабном уровне они формируют структуру, отражающую внешнее динамическое воздействие на регион. Важнейшими требованиями к модели должны быть ее применимость и возможность подтверждения или опровержения ее предсказаний на исследуемом пространственновременном масштабе, а также выполнение законов сохранения. Формальные соотношения между размерами модельного репрезентативного макрообъема и размерами блоков геосреды не являются решающим априорным аргументом в пользу принятия модели или отказа от нее.

Континуальный подход, использующий концепцию эффективной сплошной среды, для которой подробности строения на масштабе репрезентативного элемента корректно гомогенизированы, но вовсе не отброшены, зарекомендовал себя как мощный инструмент исследования дискретных и иерархических сред в различных областях физики и механики. Модели эффективной среды получаются либо континуализацией микроструктуры исходной дискретной среды, либо на основе феноменологических приближений. Успешность модели определяется тонким балансом между разумным отказом от излишних микроскопических подробностей и требованием учета принципиальных особенностей микроструктуры на макроскопическом уровне, с тем чтобы эффективная среда приобрела новые качества. Континуум Коши, в котором динамический отклик среды является локальным и описывается единственным силовым симметричным тензором второго ранга - тензором напряжений Коши T, является простейшей 3D континуальной моделью, которая, тем не менее, достаточно богата, чтобы в ее рамках при корректном учете законов сохранения вводить разнообразные соотношения, определяющие взаимосвязь Т с кинематикой деформирования. Более общие континуальные модели среды (моментные, микрополярные, микроморфные и т.д.) строятся на основе ослабления тех ограничительных предположений, которые были введены при построении континуума Коши. В частности, динамику среды могут характеризовать несимметричные тензоры силовых и моментных напряжений.

В геолого-геофизической литературе критика континуального подхода строится на том, что модель ТДТ отождествляется не просто с континуумом Коши, а с его весьма частным случаем - моделью геометрически и физически линейного изотропного однородного упругого тела. Дополнительно к этому, критики свое неумение учитывать начальные напряжения $\mathbf{T}_{0}$ представляют как де- фект модели, на основании чего заявляют, что в рамках континуального подхода невозможно моделировать энергонасыщенные среды, некорректно выписывают определяющие соотношения даже в упомянутом простейшем случае, игнорируя без соответствующих оценок не только $\mathbf{T}_{0}$, но и вращения частиц.

Необоснованная критика в адрес континуального подхода вызвана и представлениями о сплошной среде как о некоей субстанции, способной лишь пассивно реагировать на внешние усилия и, следовательно, неспособной моделировать активную геосреду. Такая критика представляется странной на фоне очевидных современных успехов континуального подхода в моделировании активных и живых систем. Часто критика основана на том, что ее авторы путают модель со способами постановки задач в рамках этой модели. Между тем, именно для геосреды более естественны постановки задач, в которых роль «граничных условий» играют данные натурных индикаторов, распределенные внутри области. Нами в тексте работы отмечается, что такие постановки уже разработаны и хорошо приспособлены для исследования активной среды.

Естественно, что континуальный подход к описанию геосреды, как и вообще любой другой подход, имеет границы своей применимости. Мы отмечаем два типа сред, для которых отсчетное описание сталкивается с принципиальными трудностями, возникающими из-за невозможности корректного выбора репрезентативного объема - лагранжева элемента эффективной среды. Во-первых, это среды с мобильными дефектами, движение которых сопровождается массообменом. Во-вторых, среды, деформирование которых происходит вследствие движений по структуре, иерархической на любом масштабном уровне вплоть до нулевого размера, в частности по фрактальной структуре. Для моделирования деформирования геосред первого типа возможно комбинирование континуального подхода с дискретным описанием движения дефектов. Моделирование геосред второго типа, по нашему мнению, пока еще ждет разработки своего подхода.

Необходимым условием корректности теории или модели является выполнение в ее рамках законов сохранения. Их игнорирование обесценивает результаты работ, если даже эти результаты удачно вписываются в существующую научную парадигму. Парадигмы в науках о твердой Земле неоднократно менялись коренным образом в прошлом, будут меняться и в будущем. Концепции Ньютона в пределах ее применимости (макроскопический масштаб описания, нерелятивистские скорости и отсутствие сильных гравитационных полей) столь 
революционные изменения не угрожают, хотя не исключено изменение интерпретации ее отдельных положений. Незыблемыми останутся симметрии пространства-времени и вытекающее отсюда существование законов сохранения. Если выбор реологической модели эффективной среды или оценка влияния предыстории деформирования на получаемый результат могут (и должны) представлять предмет для дискуссии, то выполнение законов сохранения является абсолютным и непререкаемым требованием. Их нарушение (и, главным образом, отказ от Эйлеровых законов динамики) выводит теорию из разряда физических и является неоправданно высокой ценой за мнимое понимание поведения геосреды.

В тексте нашей работы мы обсудили, почему это происходит в ряде геологических работ. Дополнительно к этому мы попытались ответить на близкие вопросы: почему в геологии термины, заимствованные из физики, искажают свой смысл и почему в рамках геологического мышления складываются благоприятные условия для того, чтобы воззрения Аристотеля и другие доньютоновские представления раз за разом возрождались и одерживали верх над идеями современной классической физики? В качестве важнейших причин мы отмечаем нацеленность геологических исследований на учет любых деталей строения геосреды (что, в конечном счете, влечет за собой неприятие модели сплошности) и ориентированность геологии на использование априорно фиксированной единой системы отсчета и выделенного класса систем координат.

Некорректность принимаемой терминологии и искажение смысла заимствованных физических терминов вносят иногда в геолого-геофизические работы путаницу и приводят к заведомо неправильным результатам. В нашей работе мы ограничились лишь несколькими примерами. Более подробно была рассмотрена проблема реконструкции напряжений по натурным кинематическим индикаторам, а именно по необратимым разрывным сдвигам. Мы обратили внимание на то, что в господствующем сейчас подходе авторы пытаются навязать геосреде некоторые априорные умозрительные правила связи искомых напряжений с кинематикой подвижек. В рамках такого подхода законы сохранения неизбежно игнорируются, что, вопреки мнению авторов, не позволяет интерпре- тировать получаемые ими результаты в терминах напряжений.

Отмечая тупиковость такого подхода, мы одновременно и уже не в первый раз предлагаем взамен подход, основанный на учете законов сохранения. Это позволит не только реконструировать поле тензора напряжений, но и, дополнительно к этому, определить диссипативную функцию (или пластический потенциал) эффективной сплошной среды, т.е. фактически выявить реологию этой среды без каких-либо предположений. Здесь уместно сослаться на афоризм Гельвеция: «Знание некоторых принципов легко возмещает незнание некоторых фактов». Роль принципов у нас играют законы сохранения, а роль фактов - связь направлений подвижек на плоскостях разрыва с действующими напряжениями. Эти факты ставят во главу угла авторы критикуемого нами подхода, не принимая во внимание того, что в каждом конкретном массиве горных пород под действием напряжений подвижки на плоскостях разрыва ориентируются так, чтобы в конечном счете удовлетворить законам сохранения и термодинамическим ограничениям, а не субъективным представлениям того или иного исследователя о том, как они должны быть ориентированы.

Резюмируя обсуждения, приведенные в тексте нашей работы, мы утверждаем, что отвергать континуальный подход априори, ссылаясь на дискретность геосреды, по меньшей мере неконструктивно. Модель сплошности - это не парадигма, а удобный рабочий инструмент для познания мира. Как и любая другая модель, она огрубляет действительность, абстрагируясь от многих несущественных, на данном уровне понимания, деталей. Однако эта модель открыта для саморазвития. Она способна инкорпорировать (за счет усложнения модели представительного элемента и динамических характеристик, за счет внесения внутренних параметров и т.д.) те детали, которые были ранее отброшены неправомерно и, на самом деле, влияют на изучаемое явление. В отдельных случаях континуальный подход нуждается в комбинировании с дискретным. Пока подход, сколько-нибудь сравнимый по общности и результативности с континуальным, не предложен. Освоение этого подхода на современном уровне является необходимым элементом в деле построения применимой и работоспособной модели геосреды.

\section{0. ЛИТЕРАTУPA / REFERENCES}

Abbott B.P. et al. (LIGO Scientific Collaboration and Virgo Collaboration), 2016. Observation of gravitational waves from a binary black hole merger. Physical Review Letters 116 (6), 061102. http://dx.doi.org/10.1103/PhysRev Lett.116.061102. 
Ambrosi D., Pettinati V., Ciarletta P., 2015. Active stress as a local regulator of global size in morphogenesis. International Journal of Non-Linear Mechanics 75, 5-14. http://dx.doi.org/10.1016/j.ijnonlinmec.2014.11.027.

Angelillo M. (Ed.), 2014. Mechanics of Masonry Structures. Springer, Vienna, 341 p.

Aristotle, 1981. Physics. In: I.D. Rozhansky (Ed.), Tractates. Four volumes. Volume 3. Mysl, Moscow, p. 59-262 (in Russian] [Аристотель. Физика // Аристотель. Сочинения. В 4-х томах. Т. 3 / Ред. И.Д. Рожанский. М.: Мысль, 1981. C. 59-262].

Avouac J.P., Tapponnier P., 1993. Kinematic model of active deformation in central Asia. Geophysical Research Letters 20 (10), 895-898. http://dx.doi.org/10.1029/93GL00128.

Bayuk I.O., 2013. Modelling reservoir rock physics: basic principles. Tekhnologii Seismorazvedki (Seismic Technology) (4), 1-27 (in Russian) [Баюк И.О. Основные принципы математического моделирования макроскопических физических свойств коллекторов углеводородов // Технологии сейсморазведки. 2013. № 4. С. 5-18].

Bažant Z.P., Yavari A., 2007. Response to A. Carpinteri, B. Chiaia, P. Cornetti and S. Puzzi's comments on "Is the cause of size effect on structural strength fractal or energetic-statistical?". Engineering Fracture Mechanics 74 (17), 28972910. http://dx.doi.org/10.1016/j.engfracmech.2007.02.026.

Belousov T.P., Mukhamediev S.A., 1990. On reconstruction of palaeostresses from rock fracturing. Izvestiya AN SSSR seriya Fizika Zemli (Izvestiya, Physics of the Solid Earth) (2), 16-29 (in Russian) [Белоусов Т.П., Мухамедиев Ш.А. К реконструкции палеонапряжений по трещиноватости горных пород // Известия АН СССР, серия Физика Земли. 1990. № 2. С. 16-29].

Belousov T.P., Mukhamediev Sh.A., Chichagov V.P., 1993. Stress-relief deformation in the epicentral zone of the 1991 Racha earthquake (Southern slope of the Greater Caucasus). Doklady AN 333 (6), 775-779 (in Russian) [Белоусов Т.П., Мухамедиев Ш.А., Чичагов В.П. Деформации разгрузки в эпицентральной зоне Рачинского землетрясения на юге Большого Кавказа // Доклады АH. 1993. Т. 333. № 6. С. 775-779].

Bott M.H.P., 1959. The mechanics of oblique slip faulting. Geological Magazine 96 (02), 109-117. http://dx.doi.org/ $10.1017 /$ S0016756800059987.

Bykov A.A., Mukhamediev Sh.A., Shishko A.N., 2014. Experimentally studying the behavior of inhomogeneities of a transparent model material under loading. Problemy Prochnosti i Plastichnosti (Problems of Strength and Plasticity) 76 (3), 243-250 (in Russian) [Быков А.А., Мухамедиев Ш.А., Шишко А.Н. Экспериментальное исследование поведения микронеоднородностей при нагружении прозрачного модельного материала // Проблемы прочности и пластичности. 2014. Вып. 76 (3). С. 243-250].

Carpinteri A., Chiaia B., Cornetti P., Puzzi S., 2007. Comments on "Is the cause of size effect on structural strength fractal or energetic-statistical?" by Bažant \& Yavari. Engineering Fracture Mechanics 74 (17), 2892-2896. http://dx. doi.org/10.1016/j.engfracmech.2007.02.006.

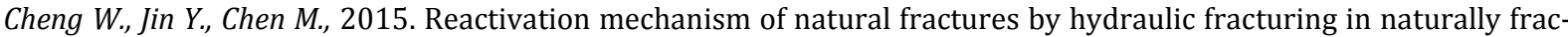
tured shale reservoirs. Journal of Natural Gas Science and Engineering 27, 1357-1365. http://dx.doi.org/10.1016/ j.jngse.2015.11.018.

Confucius, 2006. Judgments and Conversations. Phoenix, Moscow. 304 p. (in Russian) [Конфуций. Суждения и беседы. М.: Феникс, 2006. 304 с.].

Davydov D., Pelteret J.P., Steinmann P., 2014. Comparison of several staggered atomistic-to-continuum concurrent coupling strategies. Computer Methods in Applied Mechanics and Engineering 277, 260-280. http://dx.doi.org/ 10.1016/j.cma.2014.04.013.

Dell'Isola F., Maier G., Perego U., Andreaus U., Esposito R., Forest S. (Eds.), 2014. The Complete Works of Gabrio Piola, vol. I. Springer, Cham, Switzerland, 816 p.

Disy E., Garner J., 1999. Hypothetical pre-classical equations of motion. The Physics Teacher 37 (1), 42-45. http://dx. doi.org/10.1119/1.880157.

Drapaca C.S., Sivaloganathan S., 2012. A fractional model of continuum mechanics. Journal of Elasticity 107 (2), 105-123. http://dx.doi.org/10.1007/s10659-011-9346-1.

Du Bernard X., Eichhubl P., Aydin A., 2002. Dilation bands: A new form of localized failure in granular media. Geophysical Research Letters 29 (24), 2176. http://dx.doi.org/10.1029/2002GL015966.

Dyskin A.V., 2004. Continuum fractal mechanics of the Earth's crust. Pure and Applied Geophysics 161 (9-10), 19791989. http://dx.doi.org/10.1007/s00024-004-2544-2.

Engelder T., 1982. Is there a genetic relationship between selected regional joints and contemporary stress within the lithosphere of North America? Tectonics 1 (2), 161-177. http://dx.doi.org/10.1029/TC001i002p00161.

England P., Molnar P., 1997. Active deformation of Asia: from kinematics to dynamics. Science 278 (5338), 647-650. http://dx.doi.org/10.1126/science.278.5338.647.

England P., Molnar P., 2005. Late Quaternary to decadal velocity fields in Asia. Journal of Geophysical Research 110 (B12), B12401. http://dx.doi.org/10.1029/2004JB003541.

Eringen A.C., 2002. Nonlocal Continuum Field Theories. Springer, New York, 377 p.

Eshelby J.D., 1970. Energy relations and the energy-momentum tensor in continuum mechanics. In: M.F. Kanninen, W.F. Alder, A.R. Rosenfield, R.I. Joffee (Eds.), Inelastic behavior of solids. Mc Graw-Hill, New York, p. 77-115. 
Everitt C.W.F., DeBra D.B., Parkinson B.W., Turneaure J.P., Conklin J.W., Heifetz M.I., Keiser G.M., Silbergleit A.S., Holmes T., Kolodziejczak J., Al-Meshari M., Mester J.C., Muhlfelder B., Solomonik V.G., Stahl K., Worden P.W., Bencze W., Buchman S., Clarke B., Al-Jadaan A., Al-Jibreen H., Li J., Lipa J.A., Lockhart J.M., Al-Suwaidan B., Taber M., Wang S., 2011. Gravity probe B: final results of a space experiment to test general relativity. Physical Review Letters 106 (22), 221101. http://dx.doi.org/10.1103/PhysRevLett.106.221101.

Galybin A.N., Mukhamediev S.A., 2004. Determination of elastic stresses from discrete data on stress orientations. International Journal of Solids and Structures 41 (18), 5125-5142. http://dx.doi.org/10.1016/j.ijsolstr.2004.04.007.

Galybin A.N., Mukhamediev S.A., 2014. Fracture development on a weak interface ahead of a fluid-driven crack. Engineering Fracture Mechanics 129, 90-101. http://dx.doi.org/10.1016/j.engfracmech.2014.08.005.

Garagash I.A., Nikolaevskiy V.N., 2009. Cosserat mechanics in Earth sciences. Vychislitel'naya Mekhanika Sploshnykh Sred (Computational Continuum Mechanics) 2 (4), 44-66 (in Russian) [Гарагаш И.А., Николаевский В.Н. Механика Коссера для наук о Земле // Вычислительная механика сплошных сред. 2009. Т. 2. № 4. С. 44-66]. http://dx.doi.org/10.7242/1999-6691/2009.2.4.31.

Gill S.P.A., 2009. Pore migration under high temperature and stress gradients. International Journal of Heat and Mass Transfer 52 (5), 1123-1131. http://dx.doi.org/10.1016/j.ijheatmasstransfer.2008.10.004.

Ginzburg V.L., 1946. Book Review: L. Landau and E. Lifshitz. Continuum Mechanics. Gostekhizdat, Moscow, Leningrad, 1944 // Uspekhi Fizicheskikh Nauk 28 (2-3), 384-386 (in Russian) [Гинзбург В.Л. Рецензия на книгу: Л. Ландау и Е. Лифшиц. Механика сплошных сред. М.-Л.: Гостехиздат, 1944 // Успехи физических наук. 1946. Т. 28. № 2-3. C. 384-386. http://dx.doi.org/10.3367/UFNr.0028.194602k.0384].

Goldhirsch I., Goldenberg C., 2005. Stress in dense granular materials. In: H. Hinrichsen, D.E. Wolf (Eds.), The Physics of granular media. Wiley-VCH, Weinheim, p. 3-22. http://dx.doi.org/10.1002/352760362X.ch1.

Goldin S.V., 2002. Lithosphere destruction and physical mesomechanics. Fizicheskaya Mezomekhanika (Physical Mesomechanics) 5 (5), 5-20 (in Russian) [Гольдин С.В. Деструкция литосферы и физическая мезомеханика // Физическая мезомеханика. 2002. Т. 5. № 5. С. 5-22].

Grachev A.F., 2007. Regular orientation of joints in horizontally bedded sedimentary rocks of the East European platform. Izvestiya, Physics of the Solid Earth 43 (5), 360-377. http://dx.doi.org/10.1134/S1069351307050035.

Grachev A.F., Mukhamediev S.A., 2010. On the nature of the junction zone between the Vienna and Pannonian sedimentary basins. Izvestiya, Physics of the Solid Earth 46 (10), 849-861. http://dx.doi.org/10.1134/S1069351310 100058.

Gray G.G., Morgan J.K., Sanz P.F., 2014. Overview of continuum and particle dynamics methods for mechanical modeling of contractional geologic structures. Journal of Structural Geology 59, 19-36. http://dx.doi.org/10.1016/ j.jsg.2013.11.009.

Gushchenko O.I., 1973. Analysis of tectonic shear displacement orientations and their tectonophysical interpretation for reconstructions of palaeostresses. Doklady AN SSSR 210 (2), 331-334 (in Russian) [Гущенко О.И. Анализ ориентировок сколовых тектонических смещений и их тектонофизическая интерпретация при реконструкции палеонапряжений // Доклады АН СССР. 1973. Т. 210. № 2. С. 331-334].

Hancock P.L., Engelder T., 1989. Neotectonic joints. Geological Society of America Bulletin 101 (10), 1197-1208. http://dx.doi.org/10.1130/0016-7606(1989)101<1197:NJ>2.3.CO;2.

Hawkins R.J., Liverpool T.B., 2014. Stress reorganization and response in active solids. Physical Review Letters 113 (2), 028102. http://dx.doi.org/10.1103/PhysRevLett.113.028102.

Heaton T.H., 1990. Evidence for and implications of self-healing pulses of slip in earthquake rupture. Physics of the Earth and Planetary Interiors 64 (1), 1-20. http://dx.doi.org/10.1016/0031-9201(90)90002-F.

Ivlev D.D., 1966. The Theory of Ideal Plasticity. Nauka, Moscow, 232 p. (in Russian) [Ивлев Д.Д. Теория идеальной пластичности. М.: Наука, 1966. 232 с.].

Kerr R.P., 1963. Gravitational field of a spinning mass as an example of algebraically special metrics. Physical Review Letters 11 (5), 237-238. http://dx.doi.org/10.1103/PhysRevLett.11.237.

Kondaurov V.I., 1986. Energy approach to problems of continual destruction. Izvestiya AN SSSR, seriya Fizika Zemli (Izvestiya, Physics of the Solid Earth) (6), 17-22 (in Russian) [Кондауров В.И. Энергетический подход к задачам континуального разрушения // Известия АН СССР, серия Физика Земли. 1986. № 6. С. 17-22].

Kondaurov V.I., Mukhamediev Sh.A., Nikitin L.V., Ryzhak E.I., 1987. Mechanics of Rock Destruction. Nauka, Moscow, 218 p. (in Russian) [Кондауров В.И., Мухамедиев Ш.А., Никитин Л.В., Рыжак Е.И. Механика разрушения горных пород. М.: Наука, 1987. 218 с.].

Koronovskii N.V., Naimark A.A., 2015. On the continuity and discreteness of the lithosphere in the problems of dynamic geology. Moscow University Geology Bulletin 70 (1), 69-76. http://dx.doi.org/10.3103/S0145875215010020.

Koronovsky N.V., Naimark A.A., 2013. Methods of dynamic geology on critical border of applicability. Bulletin of Kamchatka Regional Association "Educational-Scientific Center". Earth Sciences (1), 152-162 (in Russian) [Короновский H.B., Наймарк A.A. Методы динамической геологии на критическом рубеже применимости // Вестник КРАУНЦ, серия Науки о Земле. 2013. № 1. С. 152-162].

Kostrov B.V., Das S., 1984. Evaluation of stress and displacement fields due to an elliptical plane shear crack. Geophysical Journal of the Royal Astronomical Society 78 (1), 19-33. http://dx.doi.org/10.1111/j.1365-246X.1984. tb06469.x. 
Kruskal M.D., 1960. Maximal extension of Schwarzschild metric. Physical Review 119 (5), 1743-1745. http://dx.doi. org/10.1103/PhysRev.119.1743.

Kuksenko V.S., Makhmudov K.F., Manzhikov B.T., 2010. Damage accumulation model for solids and the catastrophy prediction for large-scale objects. Journal of Mining Science 46 (4), 384-393. http://dx.doi.org/10.1007/s10913-0100048-z.

Kuzikov S.I., Mukhamediev S.A., 2010. Structure of the present-day velocity field of the crust in the area of the CentralAsian GPS network. Izvestiya, Physics of the Solid Earth 46 (7), 584-601. http://dx.doi.org/10.1134/S1069351 310070037.

Landau L.D., Lifshitz E.M., 1987. Theoretical Physics. 10 volumes. Volume VII. Elasticity Theory. Textbook. 4th ed., rev. and ext. Nauka, Moscow, 248 p. (in Russian) [Ландау Л.Д., Лившиц E.M. Теоретическая физика. В 10 томах. T. VII. Теория упругости: Учебное пособие. 4-е изд., испр. и доп. М.: Наука, 1987. 248 с.].

Lekhin I.V., Petrov F.N. (Eds.), 1949. Dictionary of Foreign Words, $3^{\text {rd }}$ rev. and ext. ed. GIINS, Moscow, 805 p. (in Russian) [Словарь иностранных слов. 3-е перераб. и доп. изд. / Ред. И.В. Лехин, Ф.Н. Петров М.: ГИИНС, 1949 805 c.].

Li J., Ostoja-Starzewski M., 2011. Micropolar continuum mechanics of fractal media. International Journal of Engineering Science 49 (12), 1302-1310. http://dx.doi.org/10.1016/j.ijengsci.2011.03.010.

Li S., Urata S., 2016. An atomistic-to-continuum molecular dynamics: Theory, algorithm, and applications. Computer Methods in Applied Mechanics and Engineering 306, 452-478. http://dx.doi.org/10.1016/j.cma.2016.03.048

Lin W., Conin M., Moore J.C., Chester F.M., Nakamura Y., Mori J.J., Anderson L., Brodsky E.E., Eguchi N., Cook B., Jeppson T., Wolfson-Schwehr M., Sanada Y., Saito S., Kido Y., Hirose T., Behrmann J.H., Ikari M., Ujiie K., Rowe C., Kirkpatrick J., Bose S., Regalla C., Remitti F., Toy V., Fulton P., Mishima T., Yang T., Sun T., Ishikawa T., Sample J., Takai K., Kameda J., Toczko S., Maeda L., Kodaira S., Hino R., Saffer D., 2013. Stress state in the largest displacement area of the 2011 Tohoku-Oki earthquake. Science 339 (6120), 687-690. http://dx.doi.org/10.1126/science.1229379.

Lourenço P.B., Milani G., Tralli A., Zucchini A., 2007. Analysis of masonry structures: review of and recent trends in homogenization techniques. Canadian Journal of Civil Engineering 34 (11), 1443-1457. http://dx.doi.org/10.1139/ L07-097.

Love A.E.H., 1927. A Treatise on the Mathematical Theory of Elasticity. Fourth edition. Cambridge University Press, Cambridge, 643 р. [Русский перевод: Ляв А. Математическая теория упругости (перевод с 4-го английского издания). М.-Л.: ОНТИ НКТП СССР, 1935. 674 с.].

Luk'yanov A.V., 2002. Some features of the modern tectonophysics. In: Tectonophysics today (to M.V. Gzovsky Jubilee). UIPE RAS, Moscow, p. 22-46 (in Russian) [Лукьянов А.В. Некоторые особенности современной тектонофизики // Тектонофизика сегодня (к юбилею М.В. Гзовского). М.: ОИФЗ РАН, 2002. С. 22-46].

Lurie S.Ya., 1935. Theory of Infinitesimals Used by Ancient Atomists. Publishing House of the USSR Acad. Sci., Moscow, Leningrad, 199 p. (in Russian) [Лурье С.Я. Теория бесконечно малых у древних атомистов. М.-Л.: Изд-во АН CCCP, 1935. 199 c.].

Makarov P.V., Smolin I.Yu., Stefanov Yu.P., Kuznetsov P.V., Trubitsyn A.A., Trubitsyna N.V., Voroshilov S.P., Voroshilov Ya.S., 2007. Nonlinear Geomechanics of Geomaterials and Geomedia. Geo, Novosibirsk, 235 p. (in Russian) [Макаров П.В., Смолин И.Ю., Стефанов Ю.П., Кузнецов П.В., Трубицын А.А., Трубицына Н.В., Ворошилов С.П., Ворошилов Я.С. Нелинейная геомеханика геоматериалов и геосред. Новосибирск: Гео, 2007. 235 с.].

Marchetti M.C., Joanny J.F., Ramaswamy S., Liverpool T.B., Prost J., Rao M., Simha R.A., 2013. Hydrodynamics of soft active matter. Reviews of Modern Physics 85 (3), 1143-1189. http://dx.doi.org/10.1103/RevModPhys.85.1143.

Marrett R., Peacock D.C., 1999. Strain and stress. Journal of Structural Geology 21 (8), 1057-1063. http://dx.doi.org/ 10.1016/S0191-8141(99)00020-6.

Masiani R., Trovalusci P., 1996. Cosserat and Cauchy materials as continuum models of brick masonry. Meccanica 31 (4), 421-432. http://dx.doi.org/10.1007/BF00429930.

Maugin G.A., Metrikine A.V. (Eds.), 2010. Mechanics of Generalized Continua. One Hundred Years After the Cosserats. Advances in Mechanics and Mathematics, vol. 21. Springer, New York, 337 p.

Maxwell S.C., Rutledge J., Jones R., Fehler M., 2010. Petroleum reservoir characterization using downhole microseismic monitoring. Geophysics 75 (5), 75A129-75A137. http://dx.doi.org/10.1190/1.3477966.

Mikhailov G.K., 2007. Leonhard Euler (the 300th birthday). Mekhanika Tverdogo Tela (Donetsk) 37, 3-14 (in Russian) [Михайлов Г.К. Леонард Эйлер (к 300-летию со дня рождения) // Механика твердого тела (Донецк). 2007. № 37. С. 3-14].

Mora X., 2014. Quotations on simplicity, falsifiability, the general modelling nature of mathematics, and mathematical beauty. Available from: http://mat.uab.cat/ xmora/articles/qs.pdf (last accessed June 27, 2016)

Mukhamediev S.A., 1990. Destruction Processes in the Earth's Lithosphere. Publishing House of IPE, the USSR Acad. Sci., Moscow, 204 p. (in Russian) [Мухамедиев Ш.А. Процессы разрушения в литосфере Земли. М.: Изд-во ИФЗ AH CCCP, 1990. 204 c.].

Mukhamediev S.A., 1991. Retrieving field of stress tensor in crustal blocks. Izvestiya AN SSSR, seriya Fizika Zemli (Izvestiya, Physics of the Solid Earth) (5), 29-38 (in Russian) [Мухамедиев Ш.А. К проблеме восстановления поля тензора напряжений в блоках земной коры // Известия АН СССР, серия Физика Земли. 1991. № 5. C. 29-38]. 
Mukhamediev S.A., 1993. Reconstruction of tectonic stresses on the base of slip motions data: mathematical and physical constraints. Doklady AN 331 (4), 500-503 (in Russian) [Мухамедиев Ш.A. Реконструкция тектонических напряжений по разрывным сдвиговым смещениям: математические и физические ограничения // Доклады АН. 1993. Т. 331. № 4. С. 500-503].

Mukhamediev S.A., 2002. Global stresses in the Western Europe lithosphere and the collision forces in the AfricaEurasia convergence zone. Russian Journal of Earth Sciences 4 (1), 1-17. http://dx.doi.org/10.2205/2002 ES000083.

Mukhamediev S.A., 2014. Refraction of the principal stress trajectories and the stress jumps on faults and contact surfaces: Part 1. Non-constrained regular trajectories. Izvestiya, Physics of the Solid Earth 50 (5), 655-691. http:// dx.doi.org/10.1134/S1069351314040132.

Mukhamediev S.A., 2015. What kind of information about stresses and rheology is supplied by fracture processes in the Earth's crust? Doklady Earth Sciences 461 (1), 265-269. http://dx.doi.org/10.1134/S1028334X15010055.

Mukhamediev S.A., Brady B.H.G., 2002. On methods of the macro-stress determination by fault-slip inversions. In: A.V. Dyskin, X. Hu, E. Sahouryen (Eds.), Structural integrity and fracture. Bulkema Publishers, Lisse, The Netherlands, p. 277-281.

Mukhamediev S.A., Galybin A.N., 2004. Solution of a plane elastic problem with given trajectories of the principal stresses. Doklady Physics 49 (5), 311-314. http://dx.doi.org/10.1134/1.1763623.

Mukhamediev S.A., Galybin A.N., 2007. Determination of stresses from the stress trajectory pattern in a plane elastic domain. Mathematics and Mechanics of Solids 12 (1), 75-106. http://dx.doi.org/10.1177/1081286506067093.

Mukhamediev S.A., Grachev A.F., 2000. Jointing of rocks and estimation of in situ stresses in exposures affected by explosions. Izvestiya, Physics of the Solid Earth 36 (2), 129-137.

Mukhamediev S.A., Kuzikov S.I., Zubovich A.V., 2011. Features of horizontal deformation velocity patterns and rotation in the Central Tien Shan from GPS measurement. In: Modern problems of geodynamics and geoecology of intracontinental orogens. Abstracts of the $5^{\text {th }}$ International Symposium (Bishkek, 19-24 June 2011). RS RAS, Bishkek. Vol. 2, p. 60-63 (in Russian) [Мухамедиев Ш.А., Кузиков С.И., Зубович А.В. Особенности распределения скорости горизонтальных деформаций и вращений на территории Центрального Тянь-Шаня по данным GPS-измерений // Современные проблемы геодинамики и геоэкологии внутриконтинентальных орогенов: Тезисы докладов 5-го Международного симпозиума (г. Бишкек, 19-24 июня 2011 г.). Бишкек: HC PAH, 2011. T. 2. C. 60-63].

Mukhamediev S.A., Nikitin L.V., Yunga S.L., 1976. Application of the modified method of local variations to problems of nonlinear fracture mechanics // Izvestia AN SSSR. Solid Mechanics Series 1, 76-83 (in Russian) [Мухамедиев Ш.A., Никитин Л.В., Юнга С.Л. Применение модифицированного метода локальных вариаций к задачам нелинейной механики разрушения // Известия АН СССР, серия Механика твердого тела. 1976. № 1. С. 76-83].

Mukhamediev Sh.A., Ryzhak E.I., Sinyukhina S.V., 2016. Stability of a two-layer system of inhomogeneous heavy barotropic fluids. Journal of Applied Mathematics and Mechanics (in press, available online 5 August 2016). http:// dx.doi.org/10.1016/j.jappmathmech.2016.07.005.

Mukhamediev S.A., Ul'kin D.A., 2011. Formation of systems of incompact bands parallel to the compression axis in the unconsolidated sedimentary rocks: A model. Izvestiya, Physics of the Solid Earth 47 (10), 886-901. http://dx. doi.org/10.1134/S1069351311100089.

Mukhamediev S.A., Ul'kin D.A., 2014. Micromechanics of discontinuities and high porosity bands formation in the unconsolidated sedimentary rocks. Key Engineering Materials 592-593, 133-136. http://dx.doi.org/10.4028/www. scientific.net/KEM.592-593.133.

Mukhamediev S.A., Zubovich A.V., Kuzikov S.I., 2006. Identification of crustal blocks based on GPS data. Doklady Earth Sciences 408 (1), 678-681. http://dx.doi.org/10.1134/S1028334X06040386.

Nemat-Nasser S., Hori M., 1993. Micromechanics: Overall Properties of Heterogeneous Materials. Elsevier, Amsterdam, $687 \mathrm{p}$.

Nikitin L.V., Ryzhak E.I., 1977. Regularities in destruction of rocks with internal friction and dilatancy. Izvestiya AN SSSR, seriya Fizika Zemli (Izvestiya, Physics of the Solid Earth) (5), 22-37 (in Russian) [Никитин Л.В., Рыжак Е.И. Закономерности разрушения горной породы с внутренним трением и дилатансией // Известия AH CCCP, серия Физика Земли. 1977. № 5. С. 22-37].

Nikolaevsky V.N., 1996. Geomechanics and Fluid Dynamics. Nedra, Moscow, 447 p. (in Russian) [Николаевский В.Н. Геомеханика и флюидодинамика. М.: Недра, 1996. 447 с.].

Noether E., 1918. Invariante variationsprobleme. In: Nachrichten von der Königliche Gesellschaft der Wissenschaften zu Göttingen, Mathematisch-Physikalische Klasse B. 2, s. 235-257 (in German) [Русский перевод: Нетер Э. Инвариантные вариационные задачи // Вариационные принципы механики (сборник статей классиков науки) / Ред. Л.С. Полак. М.: Физматлит, 1959. С. 611-630].

Norris J.Q., Turcotte D.L., Rundle J.B., 2015a. A damage model for fracking. International Journal of Damage Mechanics 24 (8), 1227-1238. http://dx.doi.org/10.1177/1056789515572927.

Norris J.Q., Turcotte D.L., Rundle J.B., 2015b. Anisotropy in fracking: a percolation model for observed microseismicity. Pure and Applied Geophysics 172 (1), 7-21. http://dx.doi.org/10.1007/s00024-014-0921-9. 
Nowacki W., 1970. Teoria Sprężystości. Państwowe Wydawnictwo Naukowe, Warszawa, 769 s. (in Polish) [Русский перевод: Новацкий В. Теория упругости. М.: Мир, 1975. 872 с.].

Parton V.Z., 1990. Fracture Mechanics: from Theory to Practice. Nauka, Moscow, 240 p. (in Russian) [Партон В.3. Meханика разрушения: от теории к практике. М.: Наука, 1990. 240 с.].

Peive A.V., 1961. Tectonics and magmatism. Izvestiya AN SSSR, seriya Geologicheskaya (3), 36-54 (in Russian) [Пейве A.B. Тектоника и магматизм // Известия АН СССР, серия геологическая. 1961. № 3. С. 36-54].

Pelà L., Cervera M., Roca P., 2011. Continuum damage model for orthotropic materials: application to masonry. Computer Methods in Applied Mechanics and Engineering 200 (9), 917-930. http://dx.doi.org/10.1016/j.cma.2010. 11.010.

Pollard D.D., Aydin A., 1988. Progress in understanding jointing over the past century. Geological Society of America Bulletin 100 (8), 1181-1204. http://dx.doi.org/10.1130/0016-7606(1988)100<1181:PIUJOT>2.3.CO;2.

Ponomarev V.S., 2008. Energy Capacity of Geologic Medium. Nauka, Moscow, 379 p. (in Russian) [Пономарев В.C. Энергонасыщенность геологической среды. М.: Наука, 2008. 379 с.].

Ponomarev V.S., 2011. Problems of studying an energetically active geologic medium. Geotectonics 45 (2), $157-165$. http://dx.doi.org/10.1134/S001685211102004X.

Radin C., 2008. Random close packing of granular matter. Journal of Statistical Physics 131 (4), 567-573. http:// dx.doi.org/10.1007/s10955-008-9523-1.

Rebetsky Y.L., 2013. On an missing energy source of tectonic processes. Bulletin of Kamchatka Regional Association "Educational-Scientific Center". Earth Sciences (1), 132-137 (in Russian) [Ребецкий Ю.Л. Об одном неучтенном источнике энергии тектонических процессов // Вестник КРАУНЦ. Науки о Земле. 2013. № 1. С. 132-137].

Rebetsky Y.L., 2014. Instability of layered media under gravity stress. Russian Geology and Geophysics 55 (9), $1146-$ 1152. http://dx.doi.org/10.1016/j.rgg.2014.08.010.

Revuzhenko A.F., Stazhevskii S.B., Shemyakin E.I., 1974. Mechanism of deformation of a granular material under high shear. Journal of Mining Science 10 (3), 374-377. http://dx.doi.org/10.1007/BF02509483.

Richard P., Nicodemi M., Delannay R., Ribiere P., Bideau D., 2005. Slow relaxation and compaction of granular systems. Nature Materials 4 (2), 121-128. http://dx.doi.org/10.1038/nmat1300.

Rodionov V.N., Sizov I.A., Kocharyan G.G., 1989. On modeling of natural objects in geomechanics. In: M.A. Sadovsky (Ed.), Discrete properties of geophysical medium. Nauka, Moscow, p. 14-18 (in Russian) [Родионов В.Н., Сизов И.А., Кочарян Г.Г. О моделировании природных объектов в геомеханике // Дискретные свойства геофизической среды / Ред. М.А. Садовский. М.: Наука, 1989. С. 14-18].

Rudnicki J.W., Rice J.R., 1975. Conditions for the localization of deformation in pressure-sensitive dilatant materials. Journal of the Mechanics and Physics of Solids 23 (6), 371-394. http://dx.doi.org/10.1016/0022-5096(75)90001-0.

Ryzhak E.I., 2011. Coordinateless Tensor Calculations for Continuum Mechanics. MIPT, Moscow, 170 p. (in Russian) [Рыжак Е.И. Бескоординатное тензорное исчисление для механики сплошных сред. М.: МФТИ, 2011. 170 с.].

Sadovsky M.A., 1989. On significance and meaning of discreteness in geophysics. In: M.A. Sadovsky (Ed.), Discrete Properties of Geophysical Medium. Nauka, Moscow, p. 5-14 (in Russian) [Садовский М.A. О значении и смысле дискретности в геофизике // Дискретные свойства геофизической среды / Ред. М.А. Садовский. М.: Наука, 1989. C. 5-14].

Samko S.G., Kilbas A.A., Marichev O.I., 1987. Integrals and Derivatives of Fractional Order, and Some Applications. Nauka i Tekhnika, Minsk, 688 p. (in Russian) [Самко С.Г., Килбас А.А., Маричев О.И. Интегралы и производные дробного порядка и некоторые их приложения. Минск: Наука и техника, 1987. 688 с.].

Seminsky K.Z., Kozhevnikov N.O., Cheremnykh A.V., Pospeeva E.V., Bobrov A.A., Olenchenko V.V., Tugarina M.A., Potapov V.V., Zaripov R.M., Cheremnykh A.S., 2013. Interblock zones in the crust of the southern regions of East Siberia: tectonophysical interpretation of geological and geophysical data. Geodynamics \& Tectonophysics 4 (3), 203-278 (in Russian) [Семинский К.Ж., Кожевников Н.О., Черемных А.В., Поспеева Е.В., Бобров А.А., Оленченко В.В., Тугарина M.A., Потапов В.В., Зарипов Р.М., Черемных А.C. Межблоковые зоны в земной коре юга Восточной Сибири: тектонофизическая интерпретация геолого-геофизических данных // Геодинамика и тектонофизика. 2013. T. 4. № 3. C. 203-278]. http://dx.doi.org/10.5800/GT-2013-4-3-0099.

Sherman S.I., 2012. Destruction of the lithosphere: faultblock divisibility and its tectonophysical regularities. Geody namics \& Tectonophysics 3 (4), 315-344 (in Russian) [Шерман С.И. Деструкция литосферы: разломно-блоковая делимость и ее тектонофизические закономерности // Геодинамика и тектонофизика. 2012. Т. 3. № 4. C. 315-344]. http://dx.doi.org/10.5800/GT-2012-3-4-0077.

Sherman S.I., 2015. Genetic sources and tectonophysical regularities of divisibility of the lithosphere into blocks of various ranks at different stages of its formation: tectonophysical analysis. Geodynamics \& Tectonophysics 6 (3), 387-408. http://dx.doi.org/10.5800/GT-2015-6-3-0187.

Silling S.A., Lehoucq R.B., 2008. Convergence of peridynamics to classical elasticity theory. Journal of Elasticity 93 (1), 13-37. http://dx.doi.org/10.1007/s10659-008-9163-3.

Silling S.A., Lehoucq R.B., 2010. Peridynamic theory of solid mechanics. In: H. Aref, E. van der Giessen (Eds.), Advances in applied mechanics, vol. 44, p. 73-168. http://dx.doi.org/10.1016/S0065-2156(10)44002-8. 
Sobolev G.A., Ponomarev A.V., 2003. Physics of Earthquakes and Precursors. Nauka, Moscow, 270 p. (in Russian) [Coболев Г.А., Пономарев А.В. Физика землетрясений и предвестники. М.: Наука, 2003. 270 с.].

Stoyanov S.S., 1977. Mechanism of Formation of Fracture Zones. Nedra, Moscow. 144 p. (in Russian) [Стоянов C.C. Механизм формирования разрывных зон. М.: Недра, 1977. 144 с.].

Strakhov V.N., 2007. Change of epochs in Earth sciences. Russian Journal of Earth Sciences 9 (1), ES1001. http://dx. doi.org/10.2205/2007ES000217.

Subramaniyan A.K., Sun C.T., 2008. Continuum interpretation of virial stress in molecular simulations. International Journal of Solids and Structures 45 (14), 4340-4346. http://dx.doi.org/10.1016/j.ijsolstr.2008.03.016.

Suvorov S.G., Shteinman R.Ya., 1950. For the consistently materialistic interpretation of the foundations of mechanics. Uspekhi Fizicheskikh Nauk 40 (3), 407-439 (in Russian) [Суворов С.Г., Штейнман Р.Я. За последовательноматериалистическую трактовку основ механики // Успехи физических наук. 1950. Т. 40. № 3. С. 407-439].

Takatori S.C., Brady J.F., 2016. Forces, stresses and the (thermo?) dynamics of active matter. Current Opinion in Colloid \& Interface Science 21, 24-33. http://dx.doi.org/10.1016/j.cocis.2015.12.003.

Tarasov V.E., 2015. Elasticity of fractal materials using the continuum model with non-integer dimensional space. Comptes Rendus Mécanique 343 (1), 57-73. http://dx.doi.org/10.1016/j.crme.2014.09.006.

Thatcher W., 2003. GPS constraints on the kinematics of continental deformation. International Geology Review 45 (3), 191-212. http://dx.doi.org/10.2747/0020-6814.45.3.191.

Thatcher W., 2007. Microplate model for the present-day deformation of Tibet. Journal of Geophysical Research 112 (B1), B01401. http://dx.doi.org/10.1029/2005JB004244.

Toner J., Tu Y., Ramaswamy S., 2005. Hydrodynamics and phases of flocks. Annals of Physics 318 (1), $170-244$. http://dx.doi.org/10.1016/j.aop.2005.04.011.

Torabi A., Berg S.S., 2011. Scaling of fault attributes: A review. Marine and Petroleum Geology 28 (8), $1444-1460$. http://dx.doi.org/10.1016/j.marpetgeo.2011.04.003.

Truesdell C., 1972. A First Course in Rational Continuum Mechanics. The Johns Hopkins University Press, Baltimore, Maryland [Русский перевод: Трусделл К. Первоначальный курс рациональной механики сплошных сред. М.: Мир, 1975. 592 с.].

Turcotte D.L., Moores E.M., Rundle J.B., 2014. Super fracking. Physics Today 67 (8), 34-39. http://dx.doi.org/10.1063/ PT.3.2480.

Turing A.M., 1952. The chemical basis of morphogenesis. Philosophical Transactions of the Royal Society of London B: Biological Sciences 237 (641), 37-72. http://dx.doi.org/10.1098/rstb.1952.0012.

Vikulin A.V., 2008. Physics of the Earth and Geodynamics. Textbook for geophysical courses at universities. Vitus Bering Kamchatka State University Publishing House, Petropavlovsk-Kamchatsky, 463 p. (in Russian) [Buкулин A.B. Физика Земли и геодинамика: Учебное пособие для геофизических специальностей вузов. ПетропавловскКамчатский: Изд-во КамГУ им. Витуса Беринга, 2008. 463 с.].

Vikulin A.V., 2013. Nonlinearity-fractality or rheidity-energy saturation: which categories are closer for geology? (Review on the article of N.V. Koronovskii, A.A. Naimark «Methods of dynamic geology at the critical turn of applicability»). Bulletin of Kamchatka Regional Association "Educational-Scientific Center". Earth Sciences (1), 163-168 (in Russian] [Викулин A.B. Нелинейность-фрактальность или реидность-энергонасыщенность: какие категории ближе геологии? (Отзыв на статью Н.В. Короновского, А.А. Наймарка «Методы динамической геологии на критическом рубеже применимости») // Вестник КРАУНЦ, серия Науки о Земле. 2013. № 1. C. 163-168].

Vikulin A.V., Ivanchin A.G., 2013. Modern concept of block hierarchy in the structure of geomedium and its implications in geosciences. Journal of Mining Science 49 (3), 395-408. http://dx.doi.org/10.1134/S1062739149030076.

Vikulin A.V., Makhmudov K.F., Ivanchin A.G., Gerus A.I., Dolgaya A.A., 2016. On wave and rheidity properties of the Earth's crust. Physics of the Solid State 58 (3), 561-571. http://dx.doi.org/10.1134/S1063783416030306.

Wallace R.E., 1951. Geometry of shearing stress and relation to faulting. The Journal of Geology 59 (2), $118-130$. http://dx.doi.org/10.1086/625831.

Wells D.L., Coppersmith K.J., 1994. New empirical relationships among magnitude, rupture length, rupture width, rupture area, and surface displacement. Bulletin of the Seismological Society of America 84 (4), 974-1002.

Wu H.Y., Ma K.F., Zoback M., Boness N., Ito H., Hung J.H., Hickman S., 2007. Stress orientations of Taiwan Chelungpu-Fault Drilling Project (TCDP) hole-A as observed from geophysical logs. Geophysical Research Letters 34 (1), L01303. http://dx.doi.org/10.1029/2006GL028050.

Yew C.H., Weng X., 2015. Mechanics of Hydraulic Fracturing. Second edition. Gulf Professional Publishing, Oxford, UK, $234 \mathrm{p}$.

Yuen Y.P., Kuang J.S., 2013. Fourier-based incremental homogenisation of coupled unilateral damage-plasticity model for masonry structures. International Journal of Solids and Structures 50 (20), 3361-3374. http://dx.doi.org/ 10.1016/j.ijsolstr.2013.06.001.

Zamponi F., 2008. Packings close and loose. Nature 453 (7195), 606-607. http://dx.doi.org/10.1038/453606a. 
Zavyalov A.D., 2005. From the kinetic theory of strength and fracture concentration criterion to the seismogenic fracture density and earthquake forecasting. Physics of the Solid State 47 (6), 1034-1041. http://dx.doi.org/10.1134/ 1.1946852

Zhilin P.A., 2003. Theoretical Mechanics. Fundamental Laws of Mechanics. Publishing House of the Polytechnic University, St. Petersburg, 340 p. (in Russian) [Жилин П.А. Теоретическая механика. Фундаментальные законы механики. СПб.: Изд-во Политехнического университета, 2003. 340 с.].

Zhilin P.A., 2006. Applied Mechanics. Fundamentals of the Theory of Shells. Textbook. Publishing House of the Polytechnic University, St. Petersburg. 167 p. (in Russian] [Жилин П.А. Прикладная механика. Основы теории оболочек: Учебное пособие. СПб.: Изд-во Политехнического университета, 2006. 167 с.].

Zhou M., 2003. A new look at the atomic level virial stress: on continuum-molecular system equivalence. Proceedings of the Royal Society of London A: Mathematical, Physical and Engineering Sciences 459 (2037), 2347-2392. http://dx.doi.org/10.1098/rspa.2003.1127.

Zhu H.P., Zhou Z.Y., Yang R.Y., Yu A.B., 2008. Discrete particle simulation of particulate systems: a review of major applications and findings. Chemical Engineering Science 63 (23), 5728-5770. http://dx.doi.org/10.1016/j.ces. 2008.08.006.

Zhurkov S.N., Kuksenko V.S., Petrov V.A., Saveliev V.N., Sultonov U., 1977. On forecasting of rock destruction. Izvestiya AN SSSR, seriya Fizika Zemli (Izvestiya, Physics of the Solid Earth) (6), 11-18 (in Russian) [Журков С.Н., Куксенко В.C., Петров В.А., Савельев В.Н., Султонов У. О прогнозировании разрушения горных пород // Известия АН СССР, серия Физика Земли. 1977. № 6. С. 11-18].

Zubov V.P., 1965. Development of Atomistic Concepts before the Beginning of the $21^{\text {st }}$ Century. Nauka, Moscow. 372 p. (in Russian) [Зубов В.П. Развитие атомистических представлений до начала XIX века. М.: Наука, 1965. 372 с.].

Zubovich A.V., Mukhamediev S.A., 2010. A method of superimposed triangulations for calculation of velocity gradient of horizontal movements: application to the Central Asian GPS network. Geodynamics \& Tectonophysics 1 (2), 169-185 (in Russian) [Зубович А.В., Мухамедиев Ш.А. Метод наложенных триангуляций для вычисления градиента скорости горизонтальных движений: приложение к Центрально-Азиатской GPS-сети // Геодинамика и тектонофизика. 2010. T. 1. № 2. C. 169-185]. http://dx.doi.org/10.5800/GT-2010-1-2-0013.

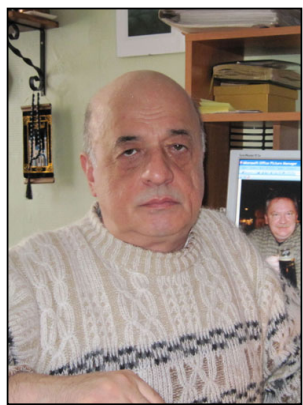

Мухамедиев Шамиль Ахмедович, докт. физ.-мат. наук, г.н.с.

Институт физики Земли им. О.Ю. Шмидта РАН

123242, ГСП-5, Москва Д-242, ул. Большая Грузинская, 10, Россия

Тел. (495)2541082,(903)1526060; \ e-mail: sh_mukhamediev@inbox.ru, shamil@ifz.ru

Mukhamediev, Shamil A., Doctor of Physics and Mathematics, Chief Researcher

O.Yu. Schmidt Institute of Physics of the Earth of RAS

10 Bol'shaya Gruzinskaya street, Moscow D-242 123242, GSP-5, Russia

Tel. (+7-495)2541082,(+7-903)1526060; \ e-mail: sh_mukhamediev@inbox.ru, shamil@ifz.ru 